BRX-TH-474

BOW-PH-119

HUTP-00/A019

\title{
Elliptic models, type IIB orientifolds, and the AdS/CFT correspondence
}

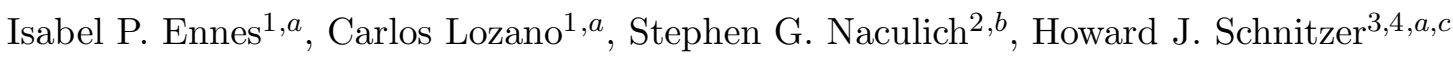 \\ ${ }^{3, a}$ Martin Fisher School of Physics \\ Brandeis University, Waltham, MA 02454 \\ ${ }^{b}$ Department of Physics \\ Bowdoin College, Brunswick, ME 04011 \\ ${ }^{c}$ Lyman Laboratory of Physics \\ Harvard University, Cambridge, MA 02138
}

\begin{abstract}
We analyze the large $N$ supergravity descriptions of the class of type IIB models T-dual to elliptic type IIA brane configurations containing two orientifold 6-planes and up to two NS 5-branes. The T-dual IIB configurations contain $N$ D3-branes in the background of an orientifold 7 -plane and, in some models, a $\mathbb{Z}_{2}$ orbifold and/or D7-branes, which give rise to four-dimensional $\mathcal{N}=2$ (or $\mathcal{N}=4$ ) gauge theories with at most two factors. We identify the chiral primary states of the supergravity theories, and match them to gauge invariant operators of the corresponding superconformal theories using Maldacena's duality.
\end{abstract}

\footnotetext{
${ }^{1}$ Research supported by the DOE under grant DE-FG02-92ER40706.

${ }^{2}$ Research supported in part by the National Science Foundation under grant no. PHY94-07194 through the ITP Scholars Program.

${ }^{3}$ Permanent address.

${ }^{4}$ Research supported in part by the DOE under grant DE-FG02-92ER40706.

naculich@bowdoin.edu; ennes,lozano,schnitzer@brandeis.edu
} 


\section{Contents}

\begin{tabular}{lll}
\hline & Introduction & 1
\end{tabular}

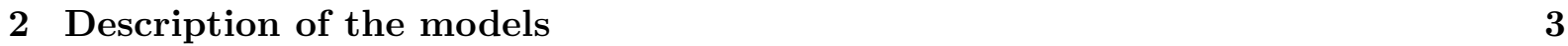

2.1 Type IIA configurations $\ldots \ldots \ldots \ldots \ldots \ldots$

2.2 Type IIB configurations $\ldots \ldots \ldots \ldots \ldots \ldots \ldots$

$\begin{array}{lll}3 & \text { Supergravity descriptions in the large } N \text { limit } & 9\end{array}$

$3.1 \mathrm{Sp}(2 N)+$ adjoint, and $\mathrm{SO}(N)+$ adjoint $\ldots \ldots \ldots \ldots \ldots$

$3.2 \mathrm{Sp}(2 N)+1$ antisymmetric +4 fundamental hypermultiplets $\ldots \ldots \ldots$. . . . . 11

$3.3 \mathrm{SU}(N)+2$ antisymmetric +4 fundamental hypermultiplets $\ldots \ldots \ldots \ldots$

$3.4 \quad \operatorname{Sp}(2 N) \times \operatorname{Sp}(2 N)+1$ bifundamental +4 fundamental hypermultiplets . . . . . 28

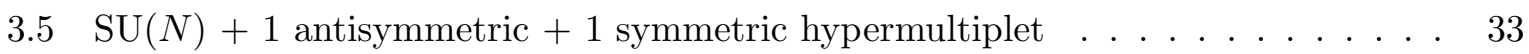

$3.6 \quad \mathrm{Sp}(2 N) \times \mathrm{SO}(2 N+2)+1$ bifundamental hypermultiplet $\ldots \ldots \ldots \ldots$

\section{Introduction}

Maldacena's duality conjecture [1]-[4] relating string theories on AdS space to conformal field theories on its boundary has proved to be extremely fruitful. The original conjecture involved the $\mathcal{N}=4$ supersymmetric $\mathrm{SU}(N)$ gauge theory on the worldvolume of $N$ D3-branes. In the large $N$ limit, the near-horizon geometry of the D3-branes is $A d S_{5} \times S^{5}$. Additional structures may be added to the background, leading to generalizations of the original proposal, where the worldvolume theory of D3-branes probing orbifold/orientifold backgrounds may be derived systematically using string-theoretic methods [5, 6].

Orbifold theories that correspond to projections of the $\mathcal{N}=4 \mathrm{SU}(N)$ theory by a discrete subgroup $\Gamma$ of the SU(4) R-symmetry group have been considered in Refs. [7]-10]. The resulting conformal field theories have either $\mathcal{N}=0,1$, or 2 supersymmetries, and the gauge group is generically of the form $\prod_{i} \mathrm{U}\left(N_{i}\right)$. They are known to be dual to IIB string theory on $A d S_{5} \times S^{5} / \Gamma$.

The near-horizon description of conformal field theories arising from D3-branes in orientifold backgrounds has been considered in Refs. [1] -[16]. The resulting field theories are dual to string 
theory on $A d S_{5} \times S^{5} / G_{\text {orient }}$, where the geometric part of the orientifold group $G_{\text {orient }}$ is again a discrete subgroup of $\mathrm{SU}(4)_{R}$.

In this paper, we analyze the large $N$ supergravity descriptions of the type IIB models T-dual to a class of elliptic type IIA brane configurations. This class of models contains two orientifold six-planes, $N$ D4-branes, (possibly) D6-branes, and at most two NS 5-branes. The T-dual IIB configurations contain $N$ D3-branes in the background of an orientifold 7-plane and (possibly) a $\mathbb{Z}_{2}$ orbifold and/or D7-branes. The four-dimensional theories arising from the worldvolume dynamics of these configurations are $\mathcal{N}=2$ ( or $\mathcal{N}=4$ ) superconformal gauge theories with simple gauge groups, or product groups with at most two factors. (The supergravity descriptions of the models in Sect. 3.1, 3.2, and 3.4 of this paper have been previously treated in Refs. [12][15], and are included here so that comparisons can be made among this entire class of elliptic models.)

In Sect. 2, we review the details of the IIA and IIB configurations for this class of theories. The large $N$ supergravity description of these models is the subject of Sect. 3 , in which we identify the spectrum of relevant and marginal chiral primary states belonging to each of these theories. The AdS/CFT correspondence is used to match the chiral primary states of the supergravity description to gauge invariant operators of the corresponding superconformal theories.

Seiberg-Witten curves [17] for the Coulomb branch of the elliptic models considered in this paper have previously been obtained using M-theory methods [18]-[22]. These constructions involve deformations of the $\mathcal{N}=4$ superconformal field theories, in which the hypermultiplets are massive. A direct connection between the Seiberg-Witten and AdS/CFT descriptions of elliptic models is not yet evident, although the AdS/CFT correspondence has recently been generalized to non-conformal theories arising from relevant deformations [23, 24]. 


\section{Description of the models}

\subsection{Type IIA configurations}

We begin by reviewing the configurations in Type IIA string theory that lead to orientifold elliptic models [18-22]. These configurations consist of NS 5-branes along 012345, D4-branes along 01236, D6-branes along 0123789, and orientifold six-planes (O6-planes) parallel to the D6-branes. The D4-branes have finite extent along $x^{6}$, which is taken to be compact. This brane configuration generically leaves $1 / 4$ of the original supersymmetries unbroken, so the effective four-dimensional theory living on the 0123 directions of the D4-branes has $\mathcal{N}=2$ supersymmetry.

Since the $x^{6}$ direction is compact, there are two O6-planes. As O6-planes carry \pm 4 units of $\mathrm{RR}$ charge (the corresponding planes will be denoted $\mathrm{O} 6^{ \pm}$), there are a priori three possibilities to consider: $\mathrm{O}^{+}-\mathrm{O}^{+}, \mathrm{O}^{-}-\mathrm{O}^{-}$, and $\mathrm{O}^{+}{ }^{-} \mathrm{O}^{-}$. The first possibility cannot be realized without breaking supersymmetry, as cancellation of RR charge in the compact direction would require the introduction of anti D6-branes; we will not consider it further. The second possibility can be realized without breaking supersymmetry, which requires the presence of 4 D6-branes (plus mirrors) to ensure the cancellation of the RR charge; this condition is equivalent to the finiteness of the low-energy field theory on the D4-branes. Finally, in the third possibility, the cancellation of RR charge (or, equivalently, finiteness of the field theory) requires the absence of D6-branes. In this paper, we will be studying two different classes of models, corresponding to the configurations $\mathrm{O}^{-}{ }^{-} \mathrm{O}^{-}$(plus D6-branes) and $\mathrm{O}^{+}{ }_{-} \mathrm{O}^{-}$.

\section{Spectra}

The spectra of the effective field theories realized on the above configurations depends critically on the number and positions of the NS 5-branes. In this work we restrict ourselves to models with at most two NS 5-branes, corresponding to elliptic models with simple gauge groups or product gauge groups with no more than two factors. 

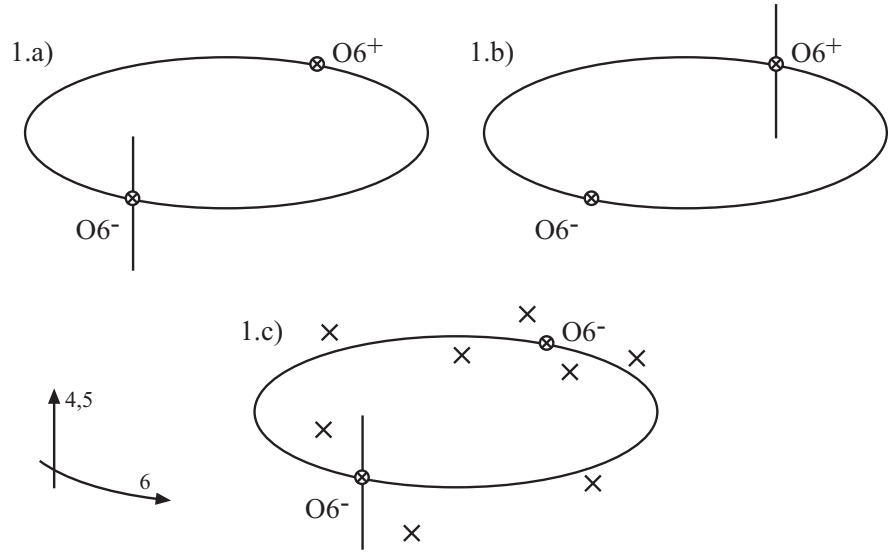

Figure 1: The three models with one NS 5-brane. The short vertical lines $\mid$ represent the NS 5-branes, the crossed circles $\otimes$ are the orientifold planes, while the crosses $\times$ denote the D6-branes, when present. D4-branes (not shown) extend along $x^{6}$. The brane configurations must be invariant under the orientifold $\mathbb{Z}_{2}$ symmetry inverting 456 .

1.a): $\mathrm{SO}(N)+$ adjoint.

1.b): $\operatorname{Sp}(2 N)+$ adjoint.

1.c): $\operatorname{Sp}(2 N)+\boxminus+4 \square$.

Figure 1 depicts the three possibilities for models with a single NS 5-brane. The $\mathrm{O}^{+}{ }^{+} \mathrm{O}^{-}$ configurations in Figs. 1.a and 1.b give rise to an $\mathcal{N}=4$ gauge theory with gauge groups $\mathrm{SO}(N)$ and $\operatorname{Sp}(2 N)$ respectively,円 or equivalently, an $\mathcal{N}=2$ vector multiplet coupled to a massless hypermultiplet in the adjoint representation of the gauge group. The $\mathrm{O}^{-}-\mathrm{O}^{-}$configuration in Fig. 1.c gives rise to an $\mathcal{N}=2$ gauge theory with gauge group $\operatorname{Sp}(2 N)$ coupled to a hypermultiplet in the antisymmetric representation $日$ and four hypermultiplets in the fundamental representation $\square$ of the gauge group.
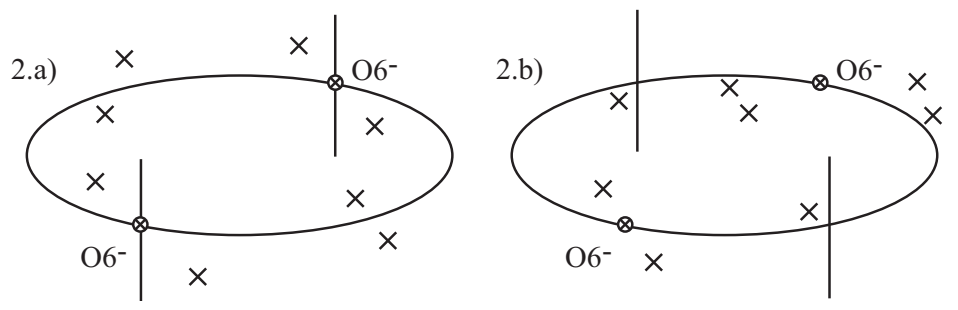

Figure 2: The two models with two NS 5-branes in an $\mathrm{O6}^{-}-\mathrm{O}^{-}$background.

2.a): $\mathrm{SU}(N)+2 \boxminus+4 \square$.

2.b): $\operatorname{Sp}(2 N)_{1} \times \operatorname{Sp}(2 N)_{2}+\left(\square_{1}, \square_{2}\right)+2 \square_{1}+2 \square_{2}$.

\footnotetext{
${ }^{1}$ Our convention is that $\operatorname{Sp}(2 N)$ has $\operatorname{rank} N$, so $\operatorname{Sp}(2) \equiv \operatorname{SU}(2)$.
} 
Figure 2 depicts models with 2 NS 5-branes in an $\mathrm{O}^{-}-\mathrm{O}^{-}$background. There are two possibilities, depending on whether the NS 5-branes intersect the O6-planes or not. If the NS 5-branes intersect the O6-planes, we have the model in Fig. 2.a, which gives rise to an $\mathcal{N}=2$ theory with gauge group $\mathrm{SU}(N)$, two hypermultiplets in the antisymmetric $\boxminus$ representation and four hypermultiplets in the fundamental $\square$ representation. On the other hand, if the NS 5-branes do not intersect the O6-planes (Fig. 2.b), we obtain an $\mathcal{N}=2$ theory with gauge group $\operatorname{Sp}(2 N) \times \operatorname{Sp}(2 N)$, a hypermultiplet in the bifundamental $(\square, \square)$ representation, and two hypermultiplets in the fundamental representation $\square$ of each of the two factors. In this case the NS 5-branes are free to move along $x^{6}$ (while respecting the symmetry $x^{6} \rightarrow-x^{6}$ ). This motion corresponds to a marginal deformation changing the relative value of coupling constants of the $\operatorname{Sp}(2 N)$ factors in the field theory on the D4-branes. Similarly, the motion of the NS 5 -brane in the (hidden) eleventh direction $x^{10}$ changes the relative value of the theta angles of the $\operatorname{Sp}(2 N)$ factors. (The average value of the coupling constants and theta angles is encoded in the M-theory description in the complex structure of the torus defined by the compact directions $x^{6}$ and $x^{10}$. Changing this complex structure - that is, the relative values of the radii $R_{6}$ and $R_{10}$ - corresponds to a second pair of marginal deformations in the field theory.)
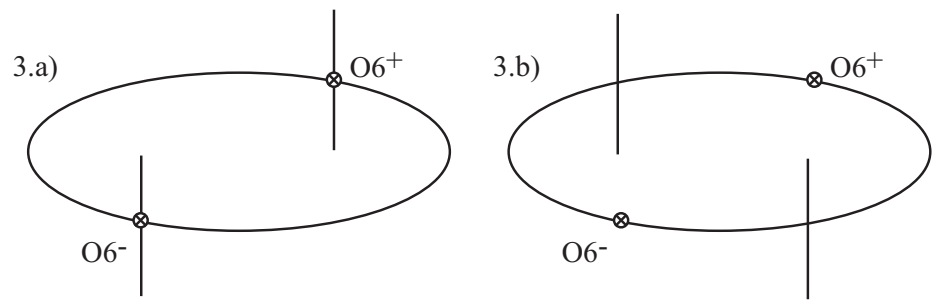

Figure 3: The two models with two NS 5-branes in an $\mathrm{O}^{+}-\mathrm{O}^{-}$background.

3.a): $\mathrm{SU}(N)+\sqcap+\square$.

3.b): $\mathrm{Sp}(2 N) \times \mathrm{SO}(2 N+2)+(\square, \square)$.

Figure 3 depicts models with 2 NS branes in an $\mathrm{O}^{+}{ }_{-}^{-} 6^{-}$background. As in the previous case, there are two possibilities to consider. The first one (Fig. 3.a) gives rise to an $\mathcal{N}=2$ theory with gauge group $\mathrm{SU}(N)$, a hypermultiplet in the symmetric representation $\square$, and a second hypermultiplet in the antisymmetric representation $\boxminus$. In the second possibility (Fig. 3.b) we obtain an $\mathcal{N}=2$ theory with gauge group $\operatorname{Sp}(2 N) \times \mathrm{SO}(2 N+2)$ and a hypermultiplet in the bifundamental $(\square, \square)$ representation, where the ranks of the factor groups are fixed by 
the finiteness of the theory. As in the $\operatorname{Sp}(2 N) \times \operatorname{Sp}(2 N)$ case, the NS 5-branes are free to move along the $x^{6}$ and $x^{10}$ directions, the motions corresponding in the field theory to marginal deformations changing the relative couplings and theta angles of the $\operatorname{Sp}(2 N)$ and $\mathrm{SO}(2 N+2)$ factors.

In all the cases discussed above, the beta functions for all the gauge groups vanish exactly.

\subsection{Type IIB configurations}

The T-dual type IIB description of the previous models has been systematically discussed by Park and Uranga [20] (see also Ref. [21]), so we will limit ourselves to a brief summary of results, and refer the reader to the above papers for further details and references. In the Tdual description, all the above models are realized as the low-energy effective theory on a stack of type IIB D3-branes probing a certain mixed orbifold-orientifold background whose details depend on the original type IIA model. As a general rule, T-duality along $x^{6}$ maps NS 5-branes stretched along 012345 to a $\mathbb{Z}_{k}^{\text {orb }}$ orbifold acting on 6789 , where $k$ is the total number of NS 5-branes (counting mirrors) present in the type IIA configuration. The O6-planes become a single O7-plane, and the D6-branes, when present, become IIB D7-branes.

Let us now consider in turn the T-dual descriptions of the type IIA configurations shown in each of the figures above.

1.a-b) For the general case of $k$ NS 5-branes in an $\mathrm{O}^{+}-\mathrm{O}^{-}$background, Park and Uranga 20] (see also Ref. 25]) have given the T-dual description as a stack of D3 branes probing an O7 background with the orientifold group

$$
G_{\text {orient }}=\mathbb{Z}_{k}^{\text {orb }}+\mathbb{Z}_{k}^{\text {orb }} \alpha \Omega^{\prime}
$$

where the $\mathbb{Z}_{k}^{\text {orb }}$ generator $\theta$ acts on $z_{1}=x^{6}+i x^{7}$ and $z_{2}=x^{8}+i x^{9}$ as

$$
z_{1} \stackrel{\theta}{\rightarrow} \mathrm{e}^{2 \pi i / k} z_{1}, \quad z_{2} \stackrel{\theta}{\rightarrow} \mathrm{e}^{-2 \pi i / k} z_{2}
$$

and $\alpha^{2}=\theta$. Also, $\Omega^{\prime}=\Omega R_{45}(-1)^{F_{L}}$, where $\Omega$ is the world-sheet parity operation, $R_{45}$ is a reflection in 45 , and $(-1)^{F_{L}}$ changes the sign of the Ramond sector of left-movers. Orientifold projections of this kind have been studied in Refs. [25]-27]. 
In figures 1.a and 1.b, there is only a single NS 5 -brane $(k=1)$, so that the $\mathbb{Z}_{k}^{\text {orb }}$ orbifold is trivial $(\theta=1)$. However, $\alpha=\theta^{1 / 2} \equiv R_{6789}$, so eq. (2.1) becomes

$$
G_{\text {orient }}=\left\{\mathbb{1}, R_{6789} \Omega^{\prime}\right\}=\left\{\mathbb{1}, \Omega R_{456789}(-1)^{F_{L}}\right\}
$$

This is actually the orientifold group of an O3-plane extending along 0123 (parallel to the probe D3-branes), precisely the setup considered in Refs. [11, 12]. Although the two different IIA configurations in figs 1.a and 1.b appear to give rise to the same IIB configuration, these configurations are actually distinguished by the background values of the two-form fields [12].

1.c) The T-dual of the configuration with $k$ NS 5-branes in an $\mathrm{O} 6^{-}-\mathrm{O}^{-}$background contains D3-branes probing an O7-plane background with the orientifold group

$$
G_{\text {orient }}=\mathbb{Z}_{k}^{\text {orb }}+\mathbb{Z}_{k}^{\text {orb }} \Omega^{\prime}
$$

with $\mathbb{Z}_{k}^{\text {orb }}$ and $\Omega^{\prime}$ as defined above [20]. Orientifold projections of this kind have been considered in $[5,25,26,28]$ for the realization of six-dimensional theories.

In figure 1.c, $k=1$, so the $\mathbb{Z}_{k}^{\text {orb }}$ orbifold is again trivial, yielding the orientifold group

$$
G_{\text {orient }}=\left\{\mathbb{1}, \Omega R_{45}(-1)^{F_{L}}\right\}
$$

which corresponds to an O7-plane extending along 01236789. The background also contains 4 D7-branes (plus mirrors) parallel to the O7-plane, which give rise to the fundamental hypermultiplets of the field theory on the probe D3-branes.

2.a-b) Figure 2 contains two NS 5-branes, so that the T-dual configuration consists of a stack of D3-branes in the background of the orientifold (2.4) with $k=2$ :

$$
G_{\text {orient }}=\mathbb{Z}_{2}^{\text {orb }}+\mathbb{Z}_{2}^{\text {orb }} \Omega^{\prime}
$$

where $\mathbb{Z}_{2}^{\text {orb }}$ acts as $\left(z_{1}, z_{2}\right) \rightarrow\left(-z_{1},-z_{2}\right)$, with $z_{1}, z_{2}$ as above. The corresponding O7-plane extends along 01236789, and the background also includes 4 D7-branes (plus mirrors). Again we find that two different IIA configurations lead apparently to the same IIB configuration. In fact, the IIB descriptions of models 2.a and 2.b are distinguished by the action of the orientifold projection $\Omega^{\prime}$ on the twisted sectors of the $\mathbb{Z}_{2}^{\text {orb }}$ orbifold, and by the action of the $\mathbb{Z}_{2}^{\text {orb }}$ orbifold projection on the D7 brane states. As pointed out in Ref. [20], and previously discussed in 
connection with Type I and six-dimensional IIB models T-dual to those considered here [28, 29, 30], $\Omega^{\prime}$ acts with an additional minus sign on the $\mathbb{Z}_{2}^{\text {orb }}$ twisted sector of model 2.b (corresponding, in the Type I context, to models with vector structure; the other possibility, leading to model 2.a, being related in the Type I context to models without vector structure [27].) Equivalently, there are two alternative (and inequivalent) choices for the Chan-Paton factors, each of which corresponds, in the type IIB context, to each of the Type IIA configurations [20].

3.a-b) In figure 3 , there are two NS branes $(k=2)$, so the orientifold group (2.1) becomes

$$
G_{\text {orient }}=\mathbb{Z}_{2}^{\text {orb }}+\mathbb{Z}_{2}^{\text {orb }} \alpha \Omega^{\prime}
$$

where $\mathbb{Z}_{2}^{\text {orb }}=\left\{\mathbb{1}, \alpha^{2}\right\}$ with $\alpha^{2}=R_{6789}$. As in the previous case, both IIA configurations lead to the same orientifold projection in the Type IIB description, but exactly as above, $\Omega^{\prime}$ acts with an additional minus sign on the $\mathbb{Z}_{2}^{\text {orb }}$ twisted sector of model 3.b.

\begin{tabular}{|c|c|c|}
\hline type IIA & $\begin{array}{c}\text { type IIB } \\
\text { Orientifold Group }\end{array}$ & Gauge theory \\
\hline $\begin{array}{l}\mathrm{O}^{+}-\mathrm{O}^{-} \text {with } 1 \mathrm{NS} 5 \\
\text { (1.a-b) }\end{array}$ & $\begin{array}{c}\left\{\mathbb{1}, R_{6789} \Omega^{\prime}\right\} \\
=\left\{\mathbb{1}, \Omega R_{456789}(-1)^{F_{L}}\right\}\end{array}$ & $\begin{array}{l}\mathrm{Sp}(2 N)+\text { adjoint } \\
\text { or } \\
\mathrm{SO}(N)+\text { adjoint }\end{array}$ \\
\hline $\begin{array}{l}\mathrm{O}^{-}-\mathrm{O}^{-} \text {with } 1 \mathrm{NS} 5 \\
\text { (1.c) }\end{array}$ & $\left\{\mathbb{1}, \Omega R_{45}(-1)^{F_{L}}\right\}$ & $\operatorname{Sp}(2 N)+\boxminus+4 \square$ \\
\hline $\begin{array}{l}\mathrm{O}^{-}-\mathrm{O}^{-} \text {with } 2 \text { NS5 } \\
(\mathbf{2 . a - b})\end{array}$ & $\mathbb{Z}_{2}^{\text {orb }}+\mathbb{Z}_{2}^{\text {orb }} \Omega^{\prime}$ & $\begin{array}{c}\mathrm{SU}(N)+2 \boxminus+4 \square \\
\text { or } \\
\operatorname{Sp}(2 N)_{1} \times \operatorname{Sp}(2 N)_{2}+\left(\square_{1}, \square_{2}\right)+2 \square_{1}+2 \square_{2}\end{array}$ \\
\hline $\begin{array}{c}\mathrm{O} 6^{+}-\mathrm{O}^{-} \text {with } 2 \mathrm{NS} 5 \\
(\mathbf{3 . a}-\mathbf{b})\end{array}$ & $\mathbb{Z}_{2}^{\text {orb }}+\mathbb{Z}_{2}^{\text {orb }} \alpha \Omega^{\prime}$ & $\begin{array}{c}\mathrm{SU}(N)+\square+\square \\
\text { or } \\
\mathrm{Sp}(2 N) \times \operatorname{SO}(2 N+2)+(\square, \square)\end{array}$ \\
\hline
\end{tabular}

Table 1: Comparative geometries. 


\section{Supergravity descriptions in the large $N$ limit}

When the number of probe D3-branes is large, the superconformal theories propagating in the world-volume of the brane configurations are conjectured to be dual to type IIB supergravity (or string theory) on a space containing an $A d S_{5}$ factor. The original formulation of the conjecture [1]- [4] related the $\mathcal{N}=4 \mathrm{SU}(N)$ gauge theory to type IIB supergravity on $A d S_{5} \times S^{5}$. The spectrum of type IIB supergravity on $A d S_{5} \times S^{5}$ falls into representations of the $\mathrm{SO}(6)$ isometry group of $S^{5}$ [31], which corresponds to the $\mathrm{SU}(4)_{R} R$-symmetry of the $\mathcal{N}=4$ theory. There are three scalar families in the supergravity spectrum containing states with zero or negative mass, summarized in Table 2 (adapted from Ref. [13]):

\begin{tabular}{|c|c|c|c|c|c|}
\hline$\Delta$ & $m^{2}$ & Range & Dynkin Label & $\mathrm{SU}(4)_{R}$ & Type IIB origin \\
\hline$k$ & $k(k-4)$ & $k \geq 2$ & $(0, k, 0)$ & $20^{\prime}, 50,105, \ldots$ & graviton +4 -form \\
\hline$k+3$ & $(k-1)(k+3)$ & $k \geq 0$ & $(0, k, 2)$ & $10,45,126, \ldots$ & 2 -forms \\
\hline$k+4$ & $k(k+4)$ & $k \geq 0$ & $(0, k, 0)$ & $\mathbf{1}, \mathbf{6}, \mathbf{2 0}$ & dilaton/axion \\
\hline
\end{tabular}

Table 2: Scalar families of IIB supergravity on $A d S_{5} \times S^{5}$ containing $m^{2} \leq 0$ states 31.

Here $m^{2}=(\Delta-p)(\Delta+p-d)$ for a $p$-form state on $A d S_{d+1}$ with $A d S$ mass $m$ coupling (in the sense of Witten [3]) to an operator of dimension $\Delta$ of the boundary $\mathcal{N}=4$ theory. There is also one family of vector fields that contains massless states (at level $k=1$ in Table 3).

\begin{tabular}{||c|c|c|c|c|c||}
\hline \hline$\Delta$ & $m^{2}$ & Range & Dynkin Label & $\mathrm{SU}(4)_{R}$ & Type IIB origin \\
\hline \hline$k+2$ & $(k-1)(k+1)$ & $k \geq 1$ & $(1, k-1,1)$ & $\mathbf{1 5 , 6 4 , \mathbf { 1 7 5 } , \ldots}$ & graviton +4 -form \\
\hline \hline
\end{tabular}

Table 3: Vector families of IIB supergravity on $A d S_{5} \times S^{5}$ containing $m^{2}=0$ states 31 .

These $k=1$ massless vector states transform in the adjoint of $\mathrm{SU}(4)_{R}$ and couple to the $\mathrm{SU}(4)_{R}$ $R$-symmetry currents (with $\Delta=3$ ) of the $\mathcal{N}=4$ theory.

In section 2 , we described a number of $\mathcal{N}=4$ or $\mathcal{N}=2$ superconformal theories propagating in the world-volume of brane configurations involving orientifold planes. These theories are 
conjectured to be dual to type IIB supergravity on $A d S_{5} \times S^{5} / G_{\text {orient }}$, where the orientifold group $G_{\text {orient }}$ depends on the specific model [11]-16] [4]. The spectrum of this supergravity background will include (i) the KK reduction of ten-dimensional type IIB theory on $A d S_{5} \times S^{5} / G_{\text {orient }}$ (obtained by a $G_{\text {orient }}$ projection of the $A d S_{5} \times S^{5}$ states), and (ii) states (invariant under $\left.G_{\text {orient }}\right)$ in sectors supported on $A d S_{5} \times Y$, where $Y \subset S^{5} / G_{\text {orient }}$ is the fixed-point set of $G_{\text {orient }}$. If the fixed-point set is empty (as, for example, in the $\mathcal{N}=4 \mathrm{SO}(N)$ and $\operatorname{Sp}(2 N)$ theories), the spectrum of the theory only contains states in (i), and is a subset of the $A d S_{5} \times S^{5}$ spectrum.

An alternative approach [14], which we will not follow in this paper, is to derive the part of the spectrum coming from the $A d S_{5}$ supergravity modes by diagonalizing the linearized equations of motion of the supergravity fields on the compact space $S^{5} / G_{\text {orient }}$. The equations for the scalar modes turn out to be Laplace equations on $S^{5} / G_{\text {orient }}$, easily written down once the corresponding metric is known. In all the cases considered in this paper, the near-horizon limit of the D3-brane metric is similar to the $A d S_{5} \times S^{5}$ solution (see for example [13], Eq. (13)), but with the metric $d \widetilde{\Omega}_{5}^{2}$ of the compact space $S^{5} / G_{\text {orient }}$ given by the angular part of

$$
d s_{S^{5} / G_{\text {orient }}}^{2}=\left|d z_{1}\right|^{2}+\left|d z_{2}\right|^{2}+|d w|^{2}=d r^{2}+r^{2} d \widetilde{\Omega}_{5}^{2}
$$

where the coordinates $z_{1}=x^{6}+i x^{7}, z_{2}=x^{8}+i x^{9}, w=x^{4}+i x^{5}$ are subject to various identifications which depend on the form of $G_{\text {orient }}$.

\section{1 $\mathrm{Sp}(2 N)+$ adjoint, and $\mathrm{SO}(N)+$ adjoint}

Let us start by reviewing the analysis of the $\mathcal{N}=4 \mathrm{SO}(N)$ and $\operatorname{Sp}(2 N)$ theories, or equivalently, $\mathcal{N}=2 \mathrm{SO}(N)$ and $\operatorname{Sp}(2 N)$ theories with massless adjoint hypermultiplets. As we saw in Sect. 2.2, the orientifold group $G_{\text {orient }}$ is that of an O3-plane acting on the $\mathbb{R}^{6}$ transverse to the D3-branes as

$$
x^{4,5,6,7,8,9} \rightarrow-x^{4,5,6,7,8,9} .
$$

In the near horizon limit the geometry becomes $A d S_{5} \times S^{5} / \mathbb{Z}_{2}$, where the geometric part of the $\mathbb{Z}_{2}$ action on $S^{5}$ is the same as the action of (3.2) on the angular part of the transverse $\mathbb{R}^{6}$. Since $S^{5}$ with such a $\mathbb{Z}_{2}$ action is actually $\mathbb{R P}^{5}$, the $\operatorname{Sp}(2 N)$ and $\operatorname{SO}(N)$ theories are dual to string theory on $A d S_{5} \times \mathbb{R P}^{5}[12]$. Further, the $\mathbb{Z}_{2}$ action has no fixed points on 
$S^{5}$, so the supergravity spectrum is simply a truncation of that for the $\mathcal{N}=4 \mathrm{SU}(N)$ theory, which can be easily determined as follows. The isometry group of $S^{5}$ is $\mathrm{SO}(6) \simeq \mathrm{SU}(4)_{R}$, which is also the $R$-symmetry group of the $\mathcal{N}=4 \mathrm{SU}(N)$ theory. The coordinates $x^{4,5,6,7,8,9}$ transform in the $\mathbf{6}$ of $\mathrm{SU}(4)_{R}$, so the $\mathbb{Z}_{2}$ action (3.2) is actually a $\mathbb{Z}_{4}$ in the center of $\mathrm{SU}(4)_{R}$ (it acts as $i \mathbb{1}$ on the 4 ). In addition, the $\Omega(-1)^{F_{L}}$ piece of the orientifold group acts with an additional minus sign on the states coming from the ten-dimensional second-rank antisymmetric tensors, leaving all other states invariant. Therefore, the spectrum consists of those states in Table 2 invariant under the combination of $\mathbb{Z}_{4}$ and $\Omega(-1)^{F_{L}}$, which are listed in Table 4 .

\begin{tabular}{||l|c|c|c|l||}
\hline \hline$\Delta$ & $m^{2}$ & Range & \multicolumn{1}{|c||}{$\mathrm{SU}(4)_{R}$} & CFT Operator \\
\hline \hline $2 k$ & $4 k(k-2)$ & $k \geq 1$ & $\mathbf{2 0}^{\prime}, \mathbf{1 0 5}, \ldots$ & $\operatorname{tr}\left(\phi^{\left\{I_{1}\right.} \cdots \phi^{\left.I_{2 k}\right\}}\right)$ \\
$2 k+3$ & $(2 k-1)(2 k+3)$ & $k \geq 0$ & $\mathbf{1 0}, \mathbf{1 2 6}, \ldots$ & $\epsilon^{\alpha \beta} \operatorname{tr}\left(\lambda_{\alpha A} \lambda_{\beta B} \phi^{\left\{I_{1}\right.} \cdots \phi^{\left.I_{2 k}\right\}}\right)+\cdots$ \\
$2 k+4$ & $4 k(k+2)$ & $k \geq 0$ & $\mathbf{1}, \mathbf{2 0}^{\prime}, \ldots$ & $\operatorname{tr}\left(F_{\mu \nu}^{2} \phi^{\left\{I_{1}\right.} \cdots \phi^{\left.I_{2 k}\right\}}\right)+\cdots$ \\
\hline \hline
\end{tabular}

Table 4: Scalars in chiral primary representations of $\mathcal{N}=4 \operatorname{Sp}(2 N)$ and $\operatorname{SO}(N)$ [12, 国. $I_{j}$ is an index in the 6 of $\mathrm{SU}(4)_{R}$, and $\operatorname{tr}\left(\phi^{\left\{I_{1}\right.} \cdots \phi^{\left.I_{2 k}\right\}}\right)$ denotes the symmetric traceless product of 6's; e.g., $\operatorname{tr}\left(\phi^{\{I} \phi^{J\}}\right)=\operatorname{tr}\left(\phi^{I} \phi^{J}\right)-\frac{1}{6} \delta^{I J} \operatorname{tr}\left(\phi^{K} \phi^{K}\right)$.

\section{2 $\mathrm{Sp}(2 N)+1$ antisymmetric +4 fundamental hypermultiplets}

The $\mathcal{N}=2 \operatorname{Sp}(2 N)$ theory with 1 antisymmetric and 4 fundamental hypermultiplets (all massless) arises as the effective theory on $N$ D3-branes probing the F-theory background first discussed by Sen [32]. This model has been much studied both from the field theory [33] 35] and dual supergravity [13, 14 viewpoints. The analysis of the AdS/CFT correspondence in Refs. [13, 14, 20] is already very complete, so we limit ourselves to a brief review of the results.

In Sect. 2.2 we found the orientifold group to be

$$
G_{\text {orient }}=\left\{\mathbb{1}, \Omega R_{45}(-1)^{F_{L}}\right\} \simeq \mathbb{Z}_{2}^{\text {orient }}
$$

where the geometric part of $\mathbb{Z}_{2}^{\text {orient }}$ acts on $w=x^{4}+i x^{5}$ as $w \rightarrow-w$. The $\mathbb{Z}_{2}^{\text {orient }}$ action fixes the hyperplane $w=0$ (which corresponds to the classical positions of the O7-plane and the D7-branes for the theory with massless fundamentals) in the six-dimensional space transverse 
to the D3-branes, which becomes $S^{3} \subset S^{5}$ in the near-horizon geometry.

In the near-horizon limit the metric for the probe D3-branes looks like $A d S_{5} \times S^{5} / \mathbb{Z}_{2}^{\text {orient }}$, with the metric of the compact space given by

$$
\begin{aligned}
& d \widetilde{\Omega}_{5}{ }^{2}=d \theta_{1}{ }^{2}+\cos ^{2} \theta_{1}\left(d \theta_{2}{ }^{2}+\cos ^{2} \theta_{2} d \phi_{1}{ }^{2}+\sin ^{2} \theta_{2} d \phi_{2}{ }^{2}\right)+\sin ^{2} \theta_{1} d \phi_{3}{ }^{2}, \\
& \theta_{1,2} \in[0, \pi / 2], \quad \phi_{1,2} \in[0,2 \pi], \quad \phi_{3} \sim \phi_{3}+\pi,
\end{aligned}
$$

where we have used the following parametrization:

$$
z_{1}=r \cos \theta_{1} \cos \theta_{2} e^{i \phi_{1}}, \quad z_{2}=r \cos \theta_{1} \sin \theta_{2} e^{i \phi_{2}}, \quad w=r \sin \theta_{1} e^{i \phi_{3}}
$$

Notice that the $\mathbb{Z}_{2}^{\text {orient }}$ orientifold action (3.3) differs from that in the $\mathcal{N}=4 \operatorname{Sp}(2 N) / \operatorname{SO}(N)$ case discussed in the previous subsection (so in this case $S^{5} / \mathbb{Z}_{2}^{\text {orient }} \nsucceq \mathbb{R P}^{5}$ ). More precisely, the isometry group of $S^{5}$ is $\mathrm{SO}(6) \supset \mathrm{SO}(4) \times \mathrm{SO}(2)$, where $\mathrm{SO}(4) \simeq \mathrm{SU}(2)_{L} \times \mathrm{SU}(2)_{R}$ is the rotation group on $\mathbb{C}^{2} \sim\left\{z_{1}, z_{2}\right\}$, and $\mathrm{SO}(2) \simeq \mathrm{U}(1)_{R}$ is the rotation group on $\mathbb{C} \sim\{w\}$. The geometric part of the orientifold group (3.3) acts only on $\mathrm{U}(1)_{R}$, identifying $e^{i \phi_{3}}$ with $e^{i\left(\phi_{3}+\pi\right)}$. On the field theory side, $\mathrm{SU}(2)_{R} \times \mathrm{U}(1)_{R} / \mathbb{Z}_{2}^{\text {orient }}$ is the $\mathcal{N}=2 R$-symmetry group, while $\mathrm{SU}(2)_{L}$ is the flavor group of the hypermultiplet in the antisymmetric representation, as we will explain further below.

The supergravity spectrum consists of (a) the $\mathbb{Z}_{2}^{\text {orient }}$ projection of the $A d S_{5} \times S^{5}$ states,

plus (b) the contribution from the fixed-point set $S^{3} \subset S^{5}$ of $\mathbb{Z}_{2}^{\text {orient }}$, which is actually the world-volume theory of $8 \mathrm{D} 7$-branes wrapping $S^{3}$ and filling the $A d S_{5}$ factor.

\section{(a) Bulk sector:}

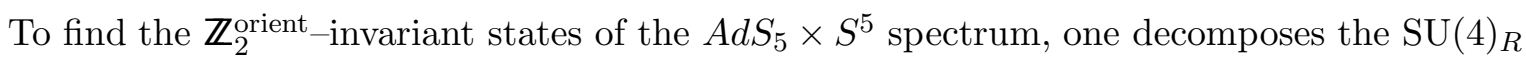
representations in Table 2 into representations $\left(d_{L}, d_{R}\right)_{q_{R}}$ of $\mathrm{SU}(2)_{L} \times \mathrm{SU}(2)_{R} \times \mathrm{U}(1)_{R}$, where $d_{L}$ and $d_{R}$ are the dimensions of the $\mathrm{SU}(2)_{L}$ and $\mathrm{SU}(2)_{R}$ representations, and $q_{R}$ is the $\mathrm{U}(1)_{R}$ charge of the state, normalized such that $w$ has $q_{R}=2$. (Table 5 lists the relevant branching rules.) Each state gets a phase $e^{i \pi q_{R} / 2}$ under $G_{\text {orient }}$, so the invariant states for representations in the first and third lines of Table 2 are those with $q_{R}=0 \bmod 4$. The orientifold acts with an additional minus sign on states in the second line of Table 2 because of the action of $\Omega(-1)^{F_{L}}$ on the ten-dimensional two-form fields. Thus, for these states, the selection rule is $q_{R}=2 \bmod 4$. 
Of the surviving states, only those satisfying the relation [36]

$$
\Delta=\frac{1}{2}\left(\left|q_{R}\right|+2\left(d_{R}-1\right)\right)
$$

where $\Delta$ is the dimension of the corresponding operator in the CFT, are (dual to) chiral primaries of the conformal field theory. The results are summarized in Table 6 .

\begin{tabular}{||l|l||}
\hline \hline $\mathrm{SU}(4)_{R}$ & $\mathrm{SU}(2)_{R} \times \mathrm{SU}(2)_{L} \times \mathrm{U}(1)_{R}$ \\
\hline \hline$(\mathbf{4})$ & $(2,1)_{ \pm 1}$ \\
$\boxminus(\mathbf{6})$ & $(1,1)_{ \pm 2}+(2,2)_{0}$ \\
$\square(\mathbf{1 0})$ & $(1,3)_{2}+(3,1)_{-2}+(2,2)_{0}$ \\
$\square(\mathbf{1 5})$ & $(1,1)_{0}+(3,1)_{0}+(1,3)_{0}+(2,2)_{ \pm 2}$ \\
$\square(\mathbf{2 0})$ & $(3,3)_{0}+(2,2)_{ \pm 2}+(1,1)_{ \pm 4}+(1,1)_{0}$ \\
$\square(\mathbf{4 5})$ & $(4,2)_{-2}+(2,4)_{2}+(2,2)_{ \pm 2}+(3,1)_{-4}+(1,3)_{4}+(3,3)_{0}+(3,1)_{0}+(1,3)_{0}$ \\
$\square(\mathbf{5 0})$ & $(4,4)_{0}+(3,3)_{ \pm 2}+(2,2)_{ \pm 4}+(2,2)_{0}+(1,1)_{ \pm 6}+(1,1)_{ \pm 2}$ \\
$\square(\mathbf{1 0 5})$ & $(5,5)_{0}+(4,4)_{ \pm 2}+(3,3)_{ \pm 4}+(3,3)_{0}+(2,2)_{ \pm 6}+(2,2)_{ \pm 2}+(1,1)_{ \pm 8}+(1,1)_{ \pm 4}+(1,1)_{0}$ \\
\hline \hline
\end{tabular}

Table 5: Branching rules for $\mathrm{SU}(4)_{R} \rightarrow \mathrm{SU}(2)_{R} \times \mathrm{SU}(2)_{L} \times \mathrm{U}(1)_{R}$.

\section{(b) D7-brane sector:}

The spectrum of chiral primaries coming from the D7-branes consists of the KK reduction of the eight-dimensional $\mathcal{N}=1$ vector multiplet [14], subject to the constraint (3.6). These states form representations of the gauge group on the D7-branes, which becomes the flavor symmetry group $\mathcal{G}_{7}$ of the hypermultiplets in the fundamental representation of the four-dimensional gauge group. In the present case $\mathcal{G}_{7}=\mathrm{SO}(8)$, as appropriate for an $\mathrm{Sp}(2 N)$ theory with four hypermultiplets in the fundamental representation. We postpone the detailed analysis of the D7-brane sector until we study the $\mathrm{SU}(N)+2 \boxminus+4 \square$ theory, and will just include the resulting chiral primary states in Table 6. 


\begin{tabular}{||c|c|c|c|c|c|c||}
\hline \hline$\Delta$ & Sector & $\mathrm{SU}(4)_{R}$ & $\left(d_{L}, d_{R}\right)_{q_{R}}$ & $\mathrm{SO}(8)_{F}$ & $m^{2}$ & $\mathrm{CFT}$ operator \\
\hline 2 & bulk & $\mathbf{2 0}^{\prime}$ & $(1,1)_{4}$ & - & -4 & $\operatorname{tr} \Phi^{2}$ \\
2 & bulk & $\mathbf{2 0}^{\prime}$ & $(3,3)_{0}$ & - & -4 & $\operatorname{tr}\left[A_{(i} J A_{j} J\right]$ \\
2 & D7 & & $(1,3)_{0}$ & 28 & -4 & $Q^{[I} J Q^{K]}$ \\
3 & bulk & $\mathbf{1 0}$ & $(1,3)_{2}$ & - & -3 & $\operatorname{tr} \lambda^{2}$ \\
3 & bulk & $\mathbf{5 0}$ & $(4,4)_{0}$ & - & -3 & $\operatorname{tr}\left[A_{(i} J A_{j} J A_{k)} J\right]$ \\
3 & bulk & $\mathbf{5 0}$ & $(2,2)_{4}$ & - & -3 & $\operatorname{tr}\left[A_{i} J \Phi^{2}\right]$ \\
3 & D7 & & $(2,4)_{0}$ & 28 & -3 & $Q^{[I} J A_{i} J Q^{K]}$ \\
4 & bulk & $\mathbf{1 0 5}$ & $(1,1)_{8}$ & - & 0 & $\operatorname{tr} \Phi^{4}$ \\
4 & bulk & $\mathbf{1 0 5}$ & $(3,3)_{4}$ & - & 0 & $\operatorname{tr}\left[A_{(i} J A_{j)} J \Phi^{2}\right]$ \\
4 & bulk & $\mathbf{1 0 5}$ & $(5,5)_{0}$ & - & 0 & $\operatorname{tr}\left[A_{(i} J A_{j} J A_{k} J A_{l)} J\right]$ \\
4 & bulk & $\mathbf{4 5}$ & $(2,4)_{2}$ & - & 0 & $\operatorname{tr}\left[\lambda^{2} A_{i} J\right]$ \\
4 & D7 & & $(3,5)_{0}$ & 28 & 0 & $Q^{[I} J A_{(i} J A_{j} J Q^{K]}$ \\
\hline \hline
\end{tabular}

Table 6 : Scalars with $\Delta \leq 4$ in chiral primary representations of $\operatorname{Sp}(2 N) \mathrm{w} / \boxminus \oplus 4 \square$. $d_{R}, d_{L}$ denote the dimensions of the $\mathrm{SU}(2)_{R}, \mathrm{SU}(2)_{L}$ representations; $q_{R}$ is the $\mathrm{U}(1)_{R}$ charge; $i, j$ are $\mathrm{SU}(2)_{L}$ indices, and $I, J$ are $\mathrm{SO}(8)_{F}$ indices.

The last column of Table 6 lists the CFT operators that correspond to the supergravity states [13, 14]. These operators are written as products of fields of the perturbative $\operatorname{Sp}(2 N)+\boxminus$ worldvolume theory, which contains an $\mathcal{N}=2$ vector multiplet in the adjoint representation, and one antisymmetric and four fundamental $\mathcal{N}=2$ hypermultiplets. Each of these $\mathcal{N}=2$ multiplets consists of several $\mathrm{SU}(2)_{R}$ representations, with definite scaling dimension $\Delta$. For example, the vector multiplet contains the scalar field $\Phi$, and the vector field $A_{\mu}$, both in $\mathrm{SU}(2)_{R}$ singlets, and the Weyl fermions $(\lambda, \psi)$ in an $\mathrm{SU}(2)_{R}$ doublet. Those $\mathrm{SU}(2)_{R}$ multiplets comprising the chiral primary operators in Table 6 are listed in Table 7 . 


\begin{tabular}{||c|c|c|c|c|}
\hline \hline$\Delta$ & $\mathrm{Sp}(2 N)$ & $\mathrm{SU}(2)_{L} \times \mathrm{SU}(2)_{R} \times \mathrm{U}(1)_{R}$ & $\mathrm{SO}(8)_{F}$ & CFT field \\
\hline \hline$\frac{3}{2}$ & adjoint & $(1,2)_{1}$ & 1 & $\lambda$ \\
1 & adjoint & $(1,1)_{2}$ & 1 & $\Phi$ \\
1 & $\boxminus$ & $(2,2)_{0}$ & 1 & $A_{i}$ \\
1 & $\square$ & $(1,2)_{0}$ & 8 & $Q^{I}$ \\
\hline \hline
\end{tabular}

Table $7: \mathrm{SU}(2)_{R}$ multiplets in perturbative $\mathrm{Sp}(2 N) \mathrm{w} / \boxminus \oplus 4 \square$

In all our tables, only the indices for the $\mathrm{SU}(2)_{L}$ and $\mathrm{SO}(8)_{F}$ symmetries are shown explicitly; the space-time, gauge, and $\mathrm{SU}(2)_{R}$ indices are implicit. The chiral primary operators are always completely symmetric in $\mathrm{SU}(2)_{R}$ indices, and only the highest component of the $\mathrm{SU}(2)_{R}$ multiplet is exhibited (e.g., $\lambda$ for the doublet $(\lambda, \psi)$ ).

The flavor symmetry of the theory is $\mathrm{SU}(2)_{L} \times \mathrm{SO}(8)$, where $\mathrm{SU}(2)_{L}$ acts only on the antisymmetric hypermultiplet, and $\mathrm{SO}(8)$ only on the four fundamental hypermultiplets. To understand this, recall that $\operatorname{Sp}(2 N)$ has only real and pseudo-real representations, which are therefore selfconjugate. If $v_{a}$ transforms in the fundamental representation of $\operatorname{Sp}(2 N)$, then $\bar{v}^{a}=J^{a b} v_{b}$ transforms in the conjugate (anti)fundamental representation, where $a, b$ are $2 N$-valued $\operatorname{Sp}(2 N)$ indices and $J^{a b}$ is the invariant antisymmetric tensor of $\operatorname{Sp}(2 N)$. $J^{a b}$ and its inverse, $J_{a b}$, are used to raise and lower $\operatorname{Sp}(2 N)$ indices. An $\mathcal{N}=2$ hypermultiplet in the antisymmetric representation of $\operatorname{Sp}(2 N)$ consists of an $\mathcal{N}=1$ chiral superfield $A_{a b}=-A_{b a}$ in the antisymmetric representation, and a second chiral superfield $\tilde{A}^{a b}=-\tilde{A}^{b a}$ in the conjugate antisymmetric representation. The piece of the $\mathcal{N}=2$ superpotential involving these fields is

$$
\tilde{A}^{a b} \Phi_{b}^{c} A_{c a}
$$

where $\Phi_{b}{ }^{c}$ is a chiral superfield in the adjoint of $\operatorname{Sp}(2 N)$. This superpotential has an obvious $U(1)_{L}$ symmetry acting as $A \rightarrow e^{i \alpha} A, \tilde{A} \rightarrow e^{-i \alpha} \tilde{A}, \Phi \rightarrow \Phi$, which is the expected flavor symmetry of a single hypermultiplet. However, as the antisymmetric representation of $\operatorname{Sp}(2 N)$ is selfconjugate, we can actually define two chiral superfields $A_{1 a b} \equiv A_{a b}$ and $A_{2 a b} \equiv J_{a c} J_{b d} \tilde{A}^{c d}$, both 
transforming in the antisymmetric representation. In terms of these new fields the superpotential reads

$$
J^{a b} A_{2 b c} \widehat{\Phi}^{c d} A_{1 d a}
$$

where $\widehat{\Phi}^{c d}=J^{a c} \Phi_{a}{ }^{d}$ is symmetric in $c, d$, as appropriate for the adjoint representation of $\operatorname{Sp}(2 N)$. Equation (3.8) is odd under exchange of $1 \leftrightarrow 2$, so the superpotential actually possesses an $\mathrm{Sp}(2) \simeq \mathrm{SU}(2)$ symmetry acting on the doublet $\left(A_{1}, A_{2}\right)$, which we can identify with the $S U(2)_{L}$ flavor symmetry that emerges from the geometry.

A similar argument holds for hypermultiplets in other representations, as follows: given $N_{k}$ hypermultiplets transforming in a $k^{\text {th }}$ rank tensor representation of $\operatorname{Sp}(2 N)$, the flavor symmetry is enhanced to $\operatorname{Sp}\left(2 N_{k}\right)$ if $k$ is even, and to $\mathrm{SO}\left(2 N_{k}\right)$ if $k$ is odd (in the latter case, the superpotential turns out to be symmetric under exchange of any pair of chiral superfields). For example, if we have $N_{f}$ hypermultiplets in the fundamental representation of $\operatorname{Sp}(2 N)$, the flavor group is $\mathrm{SO}\left(2 N_{f}\right)$. This result is actually an extension to the higher-rank case of what happens in the $\mathcal{N}=2 \mathrm{SU}(2)+N_{f} \square \simeq \mathrm{Sp}(2)+N_{f} \square$ theory discussed by Seiberg and Witten in the second paper of [17].

\section{3 $\mathrm{SU}(N)+2$ antisymmetric +4 fundamental hypermultiplets}

The $\mathcal{N}=2 \mathrm{SU}(N)$ theory with 2 antisymmetric and 4 fundamental hypermultiplets arises as the world-volume theory on $N$ D3-branes (extending along 0123) in a Type IIB brane configuration in the space-time $\mathbb{R}_{0123}^{1,3} \times \mathbb{R}_{45}^{2} / \mathbb{Z}_{2}^{\text {orient }} \times \mathbb{C}_{6789}^{2} / \mathbb{Z}_{2}^{\text {orb }}$. The orientifold group is

$$
G_{\text {orient }}=\mathbb{Z}_{2}^{\text {orb }}+\mathbb{Z}_{2}^{\text {orb }} \Omega^{\prime}=\mathbb{Z}_{2}^{\text {orb }} \times \mathbb{Z}_{2}^{\text {orient }}
$$

where $\mathbb{Z}_{2}^{\text {orb }}=\left\{\mathbb{1}, R_{6789}\right\}$ and $\mathbb{Z}_{2}^{\text {orient }}=\left\{\mathbb{1}, R_{45} \Omega(-1)^{F_{L}}\right\} . \mathbb{Z}_{2}^{\text {orient }}$ fixes the hyperplane $w=0$ (which corresponds to the classical position of the O7-plane and the 8 D7-branes, extending along 01236789), while $\mathbb{Z}_{2}^{\text {orb }}$ fixes the six-dimensional hyperplane $z_{1}=z_{2}=0$ (which corresponds to the classical position of the NS 5-branes extending along 012345 in the T-dual IIA configuration). This fixed-point set of $G_{\text {orient }}$ becomes $S^{1} \cup S^{3} \subset S^{5}$ in the near-horizon limit.

In the near horizon-limit the space-time changes to $A d S_{5} \times S^{5} /\left(\mathbb{Z}_{2}^{\text {orient }} \times \mathbb{Z}_{2}^{\text {orb }}\right)$, with the 
metric of the compact space given by

$$
\begin{gathered}
d \widetilde{\Omega}_{5}^{2}=d \theta_{1}^{2}+\cos ^{2} \theta_{1}\left(d \theta_{2}^{2}+\cos ^{2} \theta_{2} d \phi_{1}^{2}+\sin ^{2} \theta_{2} d \phi_{2}^{2}\right)+\sin ^{2} \theta_{1} d \phi_{3}{ }^{2}, \\
\theta_{1,2} \in[0, \pi], \quad \phi_{1,2,3} \sim \phi_{1,2,3}+\pi,
\end{gathered}
$$

which is the angular part of the (flat) orbifold metric

$$
d s^{2}=|d w|^{2}+\left|d z_{1}\right|^{2}+\left|d z_{2}\right|^{2}
$$

with the orbifold actions $w \stackrel{\mathbb{Z}_{2}^{\text {orient }}}{\longrightarrow}-w$ and $\left(z_{1}, z_{2}\right) \stackrel{\mathbb{Z}_{2}^{\text {orb }}}{\longrightarrow}\left(-z_{1},-z_{2}\right)$. One way to derive this metric is to observe that the T-dual of the two NS 5-branes is a two-center Taub-NUT space, which in the limit where the centers coincide becomes the orbifold $\mathbb{C}_{6789}^{2} / \mathbb{Z}_{2}^{\text {orb }}$ (see Ref. [16] for a related discussion).

The rotation group of the six-dimensional space $\mathbb{R}_{45}^{2} / \mathbb{Z}_{2}^{\text {orient }} \times \mathbb{C}_{6789}^{2} / \mathbb{Z}_{2}^{\text {orb }}$ transverse to the D3-branes is $\left[\mathrm{SU}(2)_{L} / \mathbb{Z}_{2}^{\text {orb }}\right] \times \mathrm{SU}(2)_{R} \times\left[\mathrm{U}(1)_{R} / \mathbb{Z}_{2}^{\text {orient }}\right] \subset \mathrm{SO}(6)$. This is the only part of the full global symmetry group of the worldvolume theory,

$$
\underbrace{\mathrm{SU}(2)_{R} \times \mathrm{U}(1)_{R}}_{R \text {-symmetry }} \times \underbrace{\left.\mathrm{SU}(2)_{L} \times \widetilde{\mathrm{U}(1}\right)_{L}}_{\text {flavor sym. of } 2 \square} \times \underbrace{\mathrm{SU}(4)_{F} \times \mathrm{U}(1)_{B}}_{\text {flavor sym. of } 4 \square},
$$

that is manifest in the geometric description of the brane configuration. The $\mathrm{SU}(4)_{F} \times \mathrm{U}(1)_{B}$ factor emerges as the gauge group on the D7-branes, while $\widetilde{\mathrm{U}(1)}{ }_{L}$ emerges from the low-energy dynamics of the twisted sector of $\mathbb{Z}_{2}^{\text {orb }}$.

The supergravity spectrum consists of three sectors: (a) the bulk sector, consisting of $A d S_{5} \times$ $S^{5}$ supergravity states invariant under $\mathbb{Z}_{2}^{\text {orb }} \times \mathbb{Z}_{2}^{\text {orient }},(\mathrm{b})$ the $\mathbb{Z}_{2}^{\text {orb }}$ twisted sector, supported on $A d S_{5} \times S^{1}$, consisting of the KK reduction on $S^{1}$ of the six-dimensional $(2,0)$ tensor multiplet, modded out by $\mathbb{Z}_{2}^{\text {orient }}$, and (c) the D7-brane sector, consisting of the world-volume theory of 8 D7-branes with topology $A d S_{5} \times S^{3}$, modded out by $\mathbb{Z}_{2}^{\text {orb }}$.

\section{(a) Bulk sector:}

The bulk spectrum is obtained from the IIB spectrum on $A d S_{5} \times S^{5}$ (Tables 2 and 3) by projecting onto states invariant under $\mathbb{Z}_{2}^{\text {orb }} \times \mathbb{Z}_{2}^{\text {orient }}$. The orientifold breaks $\mathrm{SO}(6) \simeq \mathrm{SU}(4)_{R} \rightarrow$ $\left[\mathrm{SU}(2)_{L} / \mathbb{Z}_{2}^{\text {orb }}\right] \times \mathrm{SU}(2)_{R} \times\left[\mathrm{U}(1)_{R} / \mathbb{Z}_{2}^{\text {orient }}\right] . \mathbb{Z}_{2}^{\text {orb }}$ coincides with the center of $\mathrm{SU}(2)_{L}$, so evendimensional representations are odd under $\mathbb{Z}_{2}^{\text {orb }}$, while odd-dimensional representations are even. 
$\mathbb{Z}_{2}^{\text {orient }}$ acts on fields with $\mathrm{U}(1)_{R}$ charge $q_{R}$ as $e^{i \pi q_{R} / 2}$, with an additional minus sign for states arising from ten-dimensional two-form fields. States must be invariant under both of these actions.

(1) Scalar modes:

Consider, for example, the supergravity scalar mode with $m^{2}=-4$ in the $\mathbf{2 0}^{\prime}$ of $\mathrm{SU}(4)_{R}$, which couples to a $\Delta=2$ operator on the boundary. Under the branching $\mathrm{SO}(6) \rightarrow \mathrm{SU}(2)_{L} \times \mathrm{SU}(2)_{R} \times$ $\mathrm{U}(1)_{R}$, this representation decomposes as (see Table 5)

$$
\mathbf{2 0}^{\prime} \rightarrow(3,3)_{0}+(2,2)_{2}+(2,2)_{-2}+(1,1)_{4}+(1,1)_{-4}+(1,1)_{0} .
$$

$\mathbb{Z}_{2}^{\text {orb }}$ projects out all the states in even-dimensional representations of $\mathrm{SU}(2)_{L}$, while only states with $q_{R}=0 \bmod 4$ survive the $\mathbb{Z}_{2}^{\text {orient }}$ projection. This leaves

$$
(3,3)_{0}+(1,1)_{4}+(1,1)_{-4}+(1,1)_{0} .
$$

Of these, only $(1,1)_{4}$ and $(3,3)_{0}$ satisfy the constraint $\Delta=\left|q_{R}\right| / 2+d_{R}-1$ and thus correspond to CFT chiral primaries with $\Delta=2$. (The state $(1,1)_{-4}$, which also satisfies the above constraint, is the complex conjugate of $(1,1)_{4}$, and is thus an antichiral primary field.)

There are two modes with $m^{2}=-3$, in the $\mathbf{1 0}$ and the $\mathbf{5 0}$ of SU(4) $R$, which couple to $\Delta=3$ operators on the boundary. The $\mathbf{5 0}$ decomposes into

$$
\mathbf{5 0} \rightarrow(4,4)_{0}+(3,3)_{ \pm 2}+(2,2)_{ \pm 4}+(2,2)_{0}+(1,1)_{ \pm 6}+(1,1)_{ \pm 2} .
$$

Again, $\mathbb{Z}_{2}^{\text {orb }}$ removes the even-dimensional representations of $\mathrm{SU}(2)_{L}$, while $\mathbb{Z}_{2}^{\text {orient }}$ only keeps $q_{R}=0 \bmod 4$, so no states survive the projection. The $\mathbf{1 0}$ decomposes into

$$
\mathbf{1 0} \rightarrow(1,3)_{2}+(3,1)_{-2}+(2,2)_{0} .
$$

Now we have to take into account the extra minus sign from the action of $\Omega(-1)^{F_{L}}$ on the ten-dimensional fields. $\mathbb{Z}_{2}^{\text {orb }}$ removes $(2,2)_{0}$, while $\mathbb{Z}_{2}^{\text {orient }}$ keeps only those states with $q_{R}=2$ $\bmod 4$. This leaves $(1,3)_{2}+(3,1)_{-2}$, of which only $(1,3)_{2}$ is chiral primary, with $\Delta=3$.

Proceeding similarly for the $\Delta=4$ states, one finds the results summarized in Table 9.

(2) Vector modes:

This set of modes is relevant because the corresponding operators couple to the conserved $\mathrm{SU}(2)_{L} \times \mathrm{SU}(2)_{R} \times \mathrm{U}(1)_{R}$ currents of the boundary CFT. The massless vector mode in the $\mathbf{1 5}$ 
of $\mathrm{SO}(6)$ (in Table 3) couples to the $\Delta=3 \mathrm{SU}(4)_{R}$ current in the $\mathcal{N}=4$ boundary theory. It decomposes into

$$
\mathbf{1 5} \rightarrow(1,1)_{0}+(2,2)_{2}+(2,2)_{-2}+(3,1)_{0}+(1,3)_{0},
$$

and, after the $G_{\text {orient }}$ projection, we end up with three currents $(1,1)_{0},(1,3)_{0},(3,1)_{0}$, none of which is a chiral primary; they are actually descendants of scalar chiral primaries. Note that $(1,1)_{0}+(1,3)_{0}$ have the correct quantum numbers to couple to the $\mathrm{SU}(2)_{R} \times \mathrm{U}(1)_{R}$ currents, while $(3,1)_{0}$ couples to the $\mathrm{SU}(2)_{L}$ current.

\section{(b) $\mathbb{Z}_{2}^{\text {orb }}$ twisted sector:}

The twisted sector of $\mathbb{Z}_{2}^{\text {orb }}$ gives rise, in the near-horizon limit, to a set of states supported on $A d S_{5} \times S^{1}$, where $S^{1} \subset S^{5}$ is left fixed by the $\mathbb{Z}_{2}^{\text {orb }}$ action. Gukov and Kapustin [15], using previous results in Ref. [10], have studied the corresponding sector of the cousin $\operatorname{Sp}(2 N) \times \operatorname{Sp}(2 N)$ theory (see Sect. 3.4 below), so we will follow their analysis closely.

Recall that the fixed-point set of $\mathbb{Z}_{2}^{\text {orb }}$, which in the near-horizon limit looks like $A d S_{5} \times S^{1}$, corresponds in the Type IIB description to the classical position of the NS 5-branes in the T-dual configuration. Accordingly, the resulting supergravity spectrum is obtained by the KK reduction on $A d S_{5} \times S^{1}$ of the six-dimensional $(2,0)$ tensor multiplet, the low-energy world-volume theory of a single NS 5-brane. In addition, one has to project the resulting states by $\mathbb{Z}_{2}^{\text {orient }}$.

Let us briefly review, following the analysis in [6], how this comes about. Start with Type IIB string theory on the orbifold $\mathbb{C}_{6789}^{2} / \mathbb{Z}_{2}^{\text {orb }}$. The massless untwisted sector contains the $(2,0)$ gravity multiplet and two tensor multiplets. There is in addition a third tensor multiplet supported on the six-dimensional hyperplane fixed by the orbifold action. The orbifold breaks the ten-dimensional transverse Lorentz group $\mathrm{SO}(8)$ to $[\mathrm{SU}(2) \times \mathrm{SU}(2)]_{\text {little group }} \times \mathrm{SU}(2)_{L} \times \mathrm{SU}(2)_{R}$, where $[\mathrm{SU}(2) \times \mathrm{SU}(2)]_{\text {little group }}$ is the little group in six dimensions and $\mathrm{SU}(2)_{L} \times \mathrm{SU}(2)_{R} \simeq \mathrm{SO}(4)$ is the rotation group in $\mathbb{C}_{6789}^{2}$. In terms of these groups, the tensor multiplet in the twisted sector contains the (bosonic) fields listed in Table 8.

\footnotetext{
${ }^{2}$ D3-branes probing this background give rise to a finite $\mathcal{N}=2 \mathrm{SU}(N) \times \mathrm{SU}(N)$ theory [8], whose dual supergravity description has been studied in Ref. [9, 10]. The IIB configuration itself is T-dual to the M-theory construction described in Ref. [18].
} 


\begin{tabular}{||c|c|c|c||}
\hline \hline Sector & {$[\mathrm{SU}(2) \times \mathrm{SU}(2)]_{\text {little group }}$} & $\mathrm{SU}(2)_{L} \times \mathrm{SU}(2)_{R}$ & 10d origin \\
\hline NS-NS & $(1,1)$ & $(1,1)$ & NS-NS 2-form \\
\hline NS-NS & $(1,1)$ & $(1,3)$ & graviton \\
\hline R-R & $(3,1)$ & $(1,1)$ & 4-form \\
\hline R-R & $(1,1)$ & $(1,1)$ & R-R 2-form \\
\hline \hline
\end{tabular}

Table 8: $(2,0)$ tensor multiplet

Now orientifold the above IIB setup by $\Omega$ only to obtain Type I on $\mathbb{C}_{6789}^{2} / \mathbb{Z}_{2}^{\text {orb }}$. The resulting Type I configuration contains an O9-plane, D9-branes (to cancel the O9 charge), and a sixdimensional plane where the $\Omega$ projection of the original IIB twisted sector propagates. This sixdimensional theory has $(1,0)$ supersymmetry, which corresponds to $\mathcal{N}=2$ in four dimensions. If we now add probe D5-branes without breaking any further supersymmetry, the resulting configuration is related by double T-duality along both $x^{4}$ and $x^{5}$ to the IIB configurations, leading to $\mathrm{SU}(N)+2 \boxminus+4 \square$ and $\operatorname{Sp}(2 N) \times \operatorname{Sp}(2 N)$.

There are two inequivalent ways to perform the $\Omega$ projection in the above setup. In the first one, leading to theories with vector structure, $\Omega$ acts with an additional minus sign on the twisted sector of $\mathbb{Z}_{2}^{\text {orb }}$. The $\Omega$ projection breaks the $(2,0)$ tensor multiplet into a $(1,0)$ tensor multiplet and a $(1,0)$ hypermultiplet, and projects out the hypermultiplet. The remaining $(1,0)$ tensor multiplet corresponds to the twisted sector of the $\operatorname{Sp}(2 N) \times \operatorname{Sp}(2 N)$ theory in the double T-dual IIB configuration. In the second possibility $\Omega$ acts without the extra minus sign (and thus projects out the $(1,0)$ tensor multiplet, keeping the hypermultiplet), leading to a model without vector structure which is T-dual to the $\mathrm{SU}(N)+2 \boxminus+4 \square$ theory.

Going back to our IIB setup, we need only perform the KK reduction of the $(2,0)$ tensor multiplet on $S^{1}$ and then project by $\mathbb{Z}_{2}^{\text {orient }}$. The reduction on $S^{1}$ gives an additional $\mathrm{U}(1)_{R}$ symmetry (coming from rotations of the circle), with a state of KK momentum $\ell \in \mathbb{Z}$ carrying $\mathrm{U}(1)_{R}$ charge $q_{R}=2 \ell$ [10, 15]. Reducing the $(2,0)$ tensor multiplet in Table 8 on $S^{1}$, we obtain the following states 10 , 15]:

(1) Scalar modes:

The triplet of scalars $(1,1 ; 1,3)$ of $[\mathrm{SU}(2) \times \mathrm{SU}(2)]_{\text {little group }} \times \mathrm{SU}(2)_{L} \times \mathrm{SU}(2)_{R}$ gives rise to a 
tower of $\mathrm{SU}(2)_{R}$ triplets $(1,3)_{2 \ell}$, where $\ell \geq 0$, with masses $m^{2}=\ell^{2}-4$, coupling to chiral operators with $\Delta=\ell+2$ and $R=2 \ell$ in the CFT. (States with $\ell<0$ couple to antichiral operators on the boundary.) $\mathbb{Z}_{2}^{\text {orient }}$ acts as $e^{i \pi q_{R} / 2}=e^{i \pi \ell}$, so only states with $\ell=0 \bmod 2$ or $q_{R}=0 \bmod 4$ survive the $\mathbb{Z}_{2}^{\text {orient }}$ projection, leaving a tower of states

$$
\begin{aligned}
\Delta=2 & (1,3)_{0}, \quad m^{2}=-4 \\
4 & (1,3)_{4}, \quad m^{2}=0
\end{aligned}
$$

These obey the constraint (3.6) and so correspond to chiral primary operators.

The pair of scalars $(1,1 ; 1,1)$ and $(1,1 ; 1,1)$ coming from the ten-dimensional 2-forms give rise to two families of KK states with quantum numbers $(1,1)_{2 \ell}$ :

(i) $m^{2}=\ell^{2}-4 \ell, \Delta=\ell, q_{R}=2 \ell, \ell \geq 2$,

(ii) $m^{2}=\ell^{2}+4 \ell, \Delta=\ell+4, q_{R}=2 \ell, \ell \geq 0$.

All the states in family (i) satisfy the constraint (3.6) and are thus potentially chiral primaries, while none of the states in family (ii) satisfy the constraint. Including the extra minus sign coming from $\Omega(-1)^{F_{L}}, \mathbb{Z}_{2}^{\text {orient }}$ acts on the states in family (i) as $e^{i \pi\left(q_{R} / 2+1\right)}=e^{i \pi(\ell+1)}$, so the projection keeps only those states with $q_{R}=2 \bmod 4$ or $\ell=1 \bmod 2$, leaving the tower of states

$$
\begin{aligned}
\Delta=3 & (1,1)_{6}, \quad m^{2}=-3 \\
5 & (1,1)_{10}, \quad m^{2}=5
\end{aligned}
$$

\section{(2) Vector modes:}

By naive dimensional reduction on $S^{1}$ we would expect to obtain a vector state from the 2-form $(3,1 ; 1,1)$. This is indeed the case [15], but only for zero KK momentum $(\ell=0)$. The resulting state is a massless vector with quantum numbers $(1,1)_{0}$ under $\mathrm{SU}(2)_{L} \times \mathrm{SU}(2)_{R} \times \mathrm{U}(1)_{R}$, which survives the $\mathbb{Z}_{2}^{\text {orient }}$ projection. This massless vector state couples to a $\Delta=3$ conserved current on the boundary, which we can identify with the $\widetilde{\mathrm{U}(1)}{ }_{L}$ current that was missing from the bulk sector. 


\section{(c) D7-brane sector:}

The AdS compactifications of Type IIB string theory T-dual to Type IIA $\mathrm{O6}^{-}-\mathrm{O6}^{-}$configurations include, as described above, 8 D7-branes with world-volume $A d S_{5} \times S^{3}$, where $S^{3} \subset S^{5} / G_{\text {orient }}$. The low-energy excitations on these 7-branes comprise a $7+1$ dimensional $\mathcal{N}=1$ vector multiplet with a gauge group $\mathcal{G}_{7} \subset \mathrm{U}(8)$ that depends on the details of $G_{\text {orient }}$. As explained in Ref. [14, the supergravity spectrum includes the KK modes of this vector multiplet on $A d S_{5} \times S^{3}$, which are dual to primary operators of the CFT charged under the global symmetry group $\mathcal{G}_{7}$.

The 7 -brane spectrum for the $\operatorname{Sp}(2 N)+\boxminus+4 \square$ theory (which has $\mathcal{G}_{7}=\mathrm{SO}(8)$ ) has been computed in Ref. [14], and for the $\operatorname{Sp}(2 N) \times \operatorname{Sp}(2 N)$ theory (which has $\mathcal{G}_{7}=\mathrm{SO}(4) \times \mathrm{SO}(4)$ ) in Ref. [15]. In the latter case, as in the $\mathrm{SU}(N)+2 \boxminus+4 \square$ theory that we are now considering (which has $\mathcal{G}_{7}=\mathrm{U}(4)$ ), the spectrum is the $\mathbb{Z}_{2}^{\text {orb }}$ projection of the $\mathcal{G}_{7}=\mathrm{SO}(8)$ case, which itself is the $\mathbb{Z}_{2}^{\text {orient }}$ projection of the $\mathcal{G}_{7}=\mathrm{U}(8)$ case (corresponding to 8 7-branes in a flat background). This comes about as follows. Start with a collection of 8 D7-branes in $\mathbb{R}^{1,9}$. As explained above, the 77 open string sector gives rise to a $7+1$ dimensional $\mathcal{N}=1$ SYM theory with gauge group $\mathcal{G}_{7}=\mathrm{U}(8)$. Now add an orientifold 7-plane parallel to the 7 -branes, which induces a $\mathbb{Z}_{2}^{\text {orient }}$ projection on the 7-brane worldvolume theory that is best described in terms of the action of the corresponding projection matrix $\gamma_{\Omega^{\prime} 7}$ on the Chan-Paton factors of the 77 strings. Gimon and Polchinski [5] have found (in the T-dual Type I setup)

$$
\gamma_{\Omega^{\prime} 7}=\mathbb{1}_{8}
$$

which enforces the projection $\mathrm{U}(8) \rightarrow \mathrm{SO}(8)$ (see, for example, [37]). To see this in more detail, let $M$ be a hermitian $8 \times 8$ Chan-Paton matrix. $\mathbb{Z}_{2}^{\text {orient }}$ enforces the projection

$$
M=-\gamma_{\Omega^{\prime} 7}^{T} M^{T} \gamma_{\Omega^{\prime} 7}^{-1}=-M^{T}
$$

from which it follows that $M$ is an antisymmetric $8 \times 8$ matrix which can be parametrized as

$$
M=\left(\begin{array}{cc}
\mathrm{a}_{1} & \mathrm{~b} \\
-\mathrm{b}^{T} & \mathrm{a}_{2}
\end{array}\right),
$$

\footnotetext{
${ }^{3}$ From the point of view of the boundary field theory, the gauge group on the 7-branes corresponds to the global flavor symmetry group of the hypermultiplets in the fundamental representation of the gauge group on the D3-branes.
} 
where $\mathrm{a}_{1}, \mathrm{a}_{2}$ are $4 \times 4$ antisymmetric matrices and $\mathrm{b}$ is a $4 \times 4$ matrix, giving a total of $6+6+16=$ 28 independent real coefficients, as appropriate for $\mathrm{SO}(8)$.

Next consider the $\mathbb{Z}_{2}^{\text {orb }}$ projection. There are two different ways of performing this projection [5], one breaking $\mathrm{SO}(8) \rightarrow \mathrm{SO}(4) \times \mathrm{SO}(4)$ (and thus corresponding to the $\mathrm{Sp}(2 N) \times \mathrm{Sp}(2 N)$ theory), the other breaking $\mathrm{SO}(8) \rightarrow \mathrm{U}(4)$ (and giving $\mathrm{SU}(N)+2 \boxminus+4 \square$ ). There are correspondingly two different projection matrices,

$$
\gamma_{\theta 7}^{\mathrm{Sp} \times \mathrm{Sp}}=\left(\begin{array}{cc}
\mathbb{1}_{4} & 0 \\
0 & -\mathbb{1}_{4}
\end{array}\right), \quad \text { and } \quad \gamma_{\theta 7}^{\mathrm{SU}}=\left(\begin{array}{cc}
0 & i \mathbb{1}_{4} \\
-i \mathbb{1}_{4} & 0
\end{array}\right)
$$

In the present case the appropriate matrix is the second one, which enforces on $M(3.22)$ the projection

$$
M=\gamma_{\theta 7}^{\mathrm{SU}} M\left(\gamma_{\theta 7}^{\mathrm{SU}}\right)^{-1} \Rightarrow M=M_{\mathrm{U}(4)}=\left(\begin{array}{cc}
\mathrm{a} & \mathrm{s} \\
-\mathrm{s} & \mathrm{a}
\end{array}\right),
$$

where $\mathrm{a}$ is a $4 \times 4$ antisymmetric matrix, and $\mathrm{S}$ is a $4 \times 4$ symmetric matrix, giving a total of $6+10=16$ real coefficients, as appropriate for $\mathrm{U}(4) \sim \mathrm{SU}(4)_{F} \times \mathrm{U}(1)_{B}$, the correct flavor group for 4 hypermultiplets in the fundamental representation of $\mathrm{SU}(N)$ (with $N \geq 3$ ).

Under the branching $\mathrm{SO}(8) \rightarrow \mathrm{SU}(4)_{F} \times \mathrm{U}(1)_{B}$ the adjoint representation of $\mathrm{SO}(8)$ decomposes as follows

$$
\mathbf{2 8} \rightarrow 1_{0}+15_{0}+6_{2}+6_{-2},
$$

where $1_{0}+15_{0}$ is the adjoint of $\mathrm{SU}(4)_{F} \times \mathrm{U}(1)_{B}$. In terms of $M(3.22)$ in the $\mathbf{2 8}$ of $\mathrm{SO}(8)$, this comes about as follows. Let $M=M_{\mathrm{U}(4)}+\widetilde{M}$, which defines $\widetilde{M}$ as a $8 \times 8$ matrix in the $6_{2} \oplus 6_{-2}$ of $\mathrm{SU}(4)_{F} \times \mathrm{U}(1)_{B}$. Then

$$
\widetilde{M}=\left(\begin{array}{cc}
\widetilde{a} & \mathrm{a}_{b} \\
-\mathrm{a}_{b}^{T} & -\widetilde{\mathrm{a}}
\end{array}\right), \quad \text { with } \quad \begin{aligned}
& \text { and } \\
& \\
&
\end{aligned}
$$

from which it is easy to see that $\left[\gamma_{\theta 7}^{\mathrm{SU}}, M_{\mathrm{U}(4)}\right]=0$ and $\left\{\gamma_{\theta 7}^{\mathrm{SU}}, \widetilde{M}\right\}=0$.

To work out the D7-brane excitations we start with the $d=8, \mathcal{N}=1$ vector multiplet in the adjoint of $\mathrm{SO}(8)$, containing a vector $A$, a complex scalar $z$, and fermions. The field $z$ is in the 
$(1,1)_{2}$ of $\mathrm{SU}(2)_{L} \times \mathrm{SU}(2)_{R} \times \mathrm{U}(1)_{R}$, where $\mathrm{U}(1)_{R}$, the rotation group of the two-dimensional space transverse to the 7 -branes, is the $R$-symmetry group in $d=8$, and $\mathrm{SU}(2)_{L} \times \mathrm{SU}(2)_{R} \simeq \mathrm{SO}(4)$ is the isometry group of $S^{3}$. Now reduce this multiplet on $A d S_{5} \times S^{3}$, to obtain [14 a KK tower of $d=5$ vector multiplets. The scalar $z$ gives rise to a tower of scalar modes in representations $(k, k)_{2}$ of $\mathrm{SU}(2)_{L} \times \mathrm{SU}(2)_{R} \times \mathrm{U}(1)_{R}$ for $k=1,2,3, \ldots$ The $d=8$ vector field $A$ decomposes into $A d S_{5}$ vectors with $\mathrm{SU}(2)_{L} \times \mathrm{SU}(2)_{R} \times \mathrm{U}(1)_{R}$ quantum numbers $(k, k)_{0}$, and two families of real scalar fields $(k, k+2)_{0} \oplus(k+2, k)_{0}$. All these states are in the adjoint representation (28) of $\mathrm{SO}(8)$. As explained in Ref. [14], they are all in short multiplets of the superconformal algebra, with their masses uniquely determined in terms of their $\mathrm{SU}(2)_{R} \times \mathrm{U}(1)_{R}$ representations. The lowest component of each such short multiplet, which is a real scalar field $(k, k+2)_{0}$ with $\Delta=k+1$, is automatically a chiral primary state of the boundary CFT.

The other components of a given short multiplet (which are protected, even though they are not chiral primaries), arise by acting with supercharges on the lowest component. The first three such multiplets (containing chiral primaries with $\Delta \leq 4$ ) are summarized in the following table:

$\mathrm{k}$

$\Delta$

2

3

4

5

6
2

$$
\begin{gathered}
(1,3)_{0}^{\text {scalar }} \\
m^{2}=-4
\end{gathered}
$$$$
\begin{gathered}
(1,1)_{2}^{\text {scalar }} \oplus(1,1)_{0}^{\text {vector }} \\
m^{2}=-3 \quad m^{2}=0
\end{gathered}
$$$$
\begin{gathered}
(2,4)_{0}^{\text {scalar }} \\
m^{2}=-3
\end{gathered}
$$$$
\begin{gathered}
(2,2)_{2}^{\text {scalar }} \oplus(2,2)_{0}^{\text {vector }} \\
m^{2}=0 \quad m^{2}=3
\end{gathered}
$$
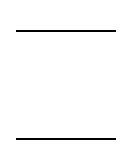

3

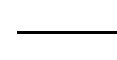

$$
(3,5)_{0}^{\text {scalar }}
$$$$
\begin{gathered}
(3,3)_{2}^{\text {scalar }} \oplus(3,3)_{0}^{\text {vector }} \\
m^{2}=5 \quad m^{2}=8
\end{gathered}
$$

$(3,1)_{0}^{\text {scalar }}$

Boxed states are chiral primaries. The massless vector $(1,1)_{0}$ in the $k=1$ family couples to the $\mathrm{SO}(8)$ current of the boundary CFT. 
We now have to project by $\mathbb{Z}_{2}^{\text {orb }}$, which acts on the above states according to their $\mathrm{SU}(2)_{L}$ representation (such that odd-dimensional representation are even, while even-dimensional representation are odd) and also as a conjugation by $\gamma_{\theta 7}^{\mathrm{SU}}$. As explained above, this breaks $\mathrm{SO}(8) \rightarrow \mathrm{U}(4) \simeq \mathrm{SU}(4)_{F} \times \mathrm{U}(1)_{B}$ and $\mathbf{2 8} \rightarrow 1_{0}+15_{0}+6_{2}+6_{-2}$. The states in the $1_{0}+15_{0}$ representation are even under conjugation by $\gamma_{\theta 7}^{\mathrm{SU}}$ (their Chan-Paton matrix $M_{\mathrm{U}(4)}$ commutes with $\gamma_{\theta 7}^{\mathrm{SU}}$ ), while the states in representations $6_{2}+6_{-2}$ are odd. Thus $\mathbb{Z}_{2}^{\text {orb }}$ keeps states in odd-dimensional representations $1,3,5, \ldots$ of $\mathrm{SU}(2)_{L}$ that transform in the adjoint $1_{0}+15_{0}$ of $\mathrm{SU}(4)_{F} \times \mathrm{U}(1)_{B}$, and states in even-dimensional representations $2,4, \cdots$ of $\mathrm{SU}(2)_{L}$ that transform in the $6_{2}+6_{-2}$ of $\mathrm{SU}(4)_{F} \times \mathrm{U}(1)_{B}$.

\begin{tabular}{||c|c|c|c|c|c|c||}
\hline \hline$\Delta$ & Sector & $\mathrm{SU}(4)_{R}$ & $\left(d_{L}, d_{R}\right)_{q_{R}}$ & $\mathrm{SU}(4)_{F} \times \mathrm{U}(1)_{B}$ & $m^{2}$ & CFT operator \\
\hline \hline 2 & bulk & $\mathbf{2 0}^{\prime}$ & $(1,1)_{4}$ & - & -4 & $\operatorname{tr} \Phi^{2}$ \\
2 & bulk & $\mathbf{2 0}^{\prime}$ & $(3,3)_{0}$ & - & -4 & $\tilde{A}_{(i} A_{j}$ \\
2 & twisted & & $(1,3)_{0}$ & - & -4 & $\tilde{A}_{[i} A_{j]}$ \\
2 & D7 & & $(1,3)_{0}$ & $1_{0} \oplus 15_{0}$ & -4 & $\tilde{Q}^{I} Q_{J}$ \\
3 & bulk & $\mathbf{1 0}$ & $(1,3)_{2}$ & - & -3 & $\operatorname{tr} \lambda \lambda$ \\
3 & twisted & & $(1,1)_{6}$ & - & -3 & $\operatorname{tr} \Phi^{3}$ \\
3 & D7 & & $(2,4)_{0}$ & $62 \oplus 6-2$ & -3 & $Q_{[I} \tilde{A}_{i} Q_{J]} \oplus \tilde{Q}^{[I} A_{i} \tilde{Q}^{J]}$ \\
4 & bulk & $\mathbf{1 0 5}$ & $(1,1)_{8}$ & - & 0 & $\operatorname{tr} \Phi^{4}$ \\
4 & bulk & $\mathbf{1 0 5}$ & $(3,3)_{4}$ & - & 0 & $\tilde{A}_{(i} \Phi^{2} A_{j)}$ \\
4 & bulk & $\mathbf{1 0 5}$ & $(5,5)_{0}$ & - & - & $\tilde{A}_{(i} A_{j} \tilde{A}_{k} A_{l)}$ \\
4 & twisted & & $(1,3)_{4}$ & - & $\tilde{A}_{[i} \Phi^{2} A_{j]}$ \\
4 & D7 & & $(3,5)_{0}$ & $1_{0} \oplus 15_{0}$ & 0 & $\tilde{Q}^{I} A_{(i} \tilde{A}_{j)} Q_{J}$ \\
\hline \hline
\end{tabular}

Table 9: Scalars with $\Delta \leq 4$ in chiral primary representations of $\mathrm{SU}(N)$ w/ $2 \boxminus \oplus 4 \square$. $d_{R}, d_{L}$ denote the dimensions of the $\mathrm{SU}(2)_{R}, \mathrm{SU}(2)_{L}$ representations; $q_{R}$ is the $\mathrm{U}(1)_{R}$ charge; $i, j$ are $\mathrm{SU}(2)_{L}$ indices, and $I, J$ are $\mathrm{SU}(4)_{F}$ indices.

The chiral primaries operators with $\Delta \leq 4$ from all the sectors are compiled in Table 9; 
anti-chiral primary operators are also present in the spectrum (and protected), and are complex conjugates of the chiral primaries. The last column of Table 9 lists the CFT operator that correspond to each supergravity state (As before, the highest component of each $\mathrm{SU}(2)_{R}$ representation is listed.) The perturbative field theory description of the worldvolume $\mathrm{SU}(N)+$ $2 \boxminus+4 \square$ theory includes an $\mathcal{N}=2$ vector multiplet in the adjoint representation and two antisymmetric and four fundamental $\mathcal{N}=2$ hypermultiplets. As described in the previous section, each of these $\mathcal{N}=2$ multiplets consists of several $\mathrm{SU}(2)_{R}$ representations, with definite scaling dimension $\Delta$. Those that comprise the chiral primary operators in Table 9 are listed in Table 10 below.

\begin{tabular}{||c|c|c|c|c|c||}
\hline \hline$\Delta$ & $\mathrm{SU}(N)$ & $\mathrm{SU}(2)_{L} \times \mathrm{SU}(2)_{R} \times \mathrm{U}(1)_{R}$ & $\widetilde{\mathrm{U}(1)_{L}}$ & $\mathrm{SU}(4)_{F} \times \mathrm{U}(1)_{B}$ & CFT field \\
\hline \hline$\frac{3}{2}$ & adjoint & $(1,2)_{1}$ & 0 & $1_{0}$ & $\lambda$ \\
1 & adjoint & $(1,1)_{2}$ & 0 & $1_{0}$ & $\Phi$ \\
1 & $\boxminus$ & $(2,2)_{0}$ & 1 & $1_{0}$ & $A_{i}$ \\
1 & $\square$ & $(1,2)_{0}$ & 0 & $4_{1}$ & $Q_{I}$ \\
\hline \hline
\end{tabular}

Table 10 : $\mathrm{SU}(2)_{R}$ multiplets in perturbative $\mathrm{SU}(N)$ w/ $2 \boxminus \oplus 4 \square$,

Before examining the table of chiral primaries further, let us consider how the flavor symmetry group $\mathrm{SU}(4)_{F} \times \mathrm{U}(1)_{B} \times \mathrm{SU}(2)_{L} \times \widetilde{\mathrm{U}(1)_{L}}$ acts on the hypermultiplet fields. The $\mathrm{SU}(4)_{F} \times \mathrm{U}(1)_{B}$ factor affects only the fundamental hypermultiplets $Q_{I}$ (and $\tilde{Q}^{I}$ in the complex conjugate representation), $I=1, \ldots, 4$, with $\mathrm{SU}(4)_{F}$ rotating the $I$ index, so that $Q_{I}\left(\tilde{Q}^{I}\right)$ transforms in the $4(\overline{4})$, and with $\mathrm{U}(1)_{B}$ acting as

$$
Q_{I} \rightarrow e^{i \alpha} Q_{I}, \quad \tilde{Q}^{J} \rightarrow e^{-i \alpha} \tilde{Q}^{J},
$$

The $\mathrm{SU}(2)_{L} \times \widetilde{\mathrm{U}(1)_{L}}$ factor acts only on the two antisymmetric hypermultiplets, each of which comprises a pair of $\mathcal{N}=1$ chiral superfields in the antisymmetric and the complex conjugate

\footnotetext{
${ }^{4}$ The identification of the operator corresponding to the $(1,3)_{4}$ state is not completely unambiguous, as tr
} $\lambda \lambda \Phi$ has the same quantum numbers. 
antisymmetric representations respectively, which in obvious notation are

$$
\left\{A_{1}, \tilde{A}^{1}\right\},\left\{A_{2}, \tilde{A}^{2}\right\}
$$

Now $\left(A_{1}, A_{2}\right)$ form a $\left.\mathrm{U}(2)_{L} \sim \mathrm{SU}(2)_{L} \times \widetilde{\mathrm{U}(1}\right)_{L}$ doublet, whereas $\left(A_{1},\left(\tilde{A}^{1}\right)^{\dagger}\right)$ and $\left(A_{2},\left(\tilde{A}^{2}\right)^{\dagger}\right)$ form $\mathrm{SU}(2)_{R}$ doublets. $\mathrm{U}(2)_{L}$ is a flavor symmetry for the 2 antisymmetric hypermultiplets, as can be seen from the piece of the tree-level superpotential

$$
\mathcal{W}=\operatorname{tr}\left(\tilde{A}^{1} \Phi A_{1}+\tilde{A}^{2} \Phi A_{2}\right)
$$

involving the antisymmetric hypermultiplets. (As we are considering the semiclassical limit of the $\mathcal{N}=2$ theory, we need not worry about the fate of the superpotential in the strong-coupling regime.) $\mathrm{SU}(2)_{L}$ arises as a geometric symmetry in the $A d S$ picture, while $\widetilde{\mathrm{U}(1)_{L}}$ corresponds to the abelian massless vector we found in the twisted sector of the $\mathbb{Z}_{2}^{\text {orb }}$.

We can give tree-level mass terms to the 2 antisymmetric hypermultiplets as follows:

$$
m_{1} \tilde{A}^{1} A_{1}+m_{2} \tilde{A}^{2} A_{2}
$$

If $m_{1} \neq m_{2}, \mathrm{U}(2)_{L}$ is broken to $\mathrm{U}(1)_{L} \times \widetilde{\mathrm{U}(1)_{L}}$, where each of the $\mathrm{U}(1)$ 's acts as follows

$$
\begin{aligned}
& \mathrm{U}(1)_{L} \quad \widetilde{\mathrm{U}(1)_{L}} \\
& A_{1} \rightarrow e^{i \alpha} A_{1} \quad \tilde{A}^{1} \rightarrow e^{-i \alpha} \tilde{A}^{1} \quad A_{1} \rightarrow e^{i \beta} A_{1} \quad \tilde{A}^{1} \rightarrow e^{-i \beta} \tilde{A}^{1} \\
& A_{2} \rightarrow e^{-i \alpha} A_{2} \quad \tilde{A}^{2} \rightarrow e^{i \alpha} \tilde{A}^{2} \quad A_{2} \rightarrow e^{i \beta} A_{2} \quad \tilde{A}^{2} \rightarrow e^{-i \beta} \tilde{A}^{2}
\end{aligned}
$$

If $m_{1}=m_{2}$, the full $\mathrm{U}(2)_{L}$ is restored. To see this, define $\mathrm{m}=\left(m_{1}-m_{2}\right) / 2$ (known as the global mass in the M-theory description [18, 19, 22]) and $\mu=\left(m_{1}+m_{2}\right) / 2$ so that $(3.30)$ becomes

$$
\begin{aligned}
& \mathrm{m}\left(\tilde{A}^{1} A_{1}-\tilde{A}^{2} A_{2}\right)+\mu\left(\tilde{A}^{1} A_{1}+\tilde{A}^{2} A_{2}\right) \\
= & \mathrm{m}\left(\tilde{A}_{2} A_{1}+\tilde{A}_{1} A_{2}\right)+\mu\left(\tilde{A}_{2} A_{1}-\tilde{A}_{1} A_{2}\right),
\end{aligned}
$$

where in the last line, we have lowered the $\mathrm{SU}(2)_{L}$ indices with the $\epsilon$ tensor.

Returning to Table 9, we find two relevant and one marginal deformations corresponding to the Coulomb branch moduli (the Casimirs tr $\Phi^{n}$, with $n \leq 4$ ), and a relevant $\Delta=3$ deformation corresponding to the gaugino condensate. We also find three relevant $\Delta=2$ deformations containing the mass operators of the two antisymmetric hypermultiplets and the mass operator 
of the fundamental hypermultiplets. Comparing eq. (3.33) with Table 9, we observe that the $(3,3)_{0}$ operator arising from the bulk supergravity sector corresponds to a deformation of the global mass $\mathrm{m}$, whereas the $(1,3)_{0}$ operator arising from the twisted sector corresponds to a deformation of the average mass $\mu$ of the antisymmetric hypermultiplets. From field theory considerations, we should expect to find at least two marginal deformations with zero $R$-charge corresponding to the change of the gauge coupling and theta angle. Actually, these deformations correspond to a CFT complex scalar operator $\operatorname{tr}\left(F^{2}+i F * F\right)$, which is a descendant (hence not a chiral primary) of $\operatorname{tr} \Phi^{2}$. On the supergravity side, this state is a singlet of $\operatorname{SU}(4)_{R}$ with $\Delta=4$ and $m^{2}=0$, which survives the $G_{\text {orient }}$ projection but is not a chiral primary.

Finally, Table 11 contains a summary of the massless vector states we have found, which couple to the $\Delta=3$ (conserved) currents of the full global symmetry group. Notice that a $(1,1)_{0}$ massless vector state in the adjoint of $\mathrm{SU}(4)_{F} \times \mathrm{U}(1)_{B}$ in the D7-brane sector survives the $\mathbb{Z}_{2}^{\text {orb }}$ projection, and couples to the $\mathrm{SU}(4)_{F} \times \mathrm{U}(1)_{B}$ flavor current of the boundary CFT.

\begin{tabular}{||c|c|c|c|c|c|c||}
\hline \hline$\Delta$ & Sector & $\mathrm{SU}(4)_{R}$ & $\left(d_{L}, d_{R}\right)_{q_{R}}$ & $\mathrm{SU}(4)_{F} \times \mathrm{U}(1)_{B}$ & $m^{2}$ & CFT current \\
\hline \hline 3 & bulk & $\mathbf{1 5}$ & $(1,3)_{0}$ & - & 0 & $\mathrm{SU}(2)_{R}$ \\
3 & bulk & $\mathbf{1 5}$ & $(1,1)_{0}$ & - & 0 & $\mathrm{U}(1)_{R}$ \\
3 & bulk & $\mathbf{1 5}$ & $(3,1)_{0}$ & - & 0 & $\mathrm{SU}(2)_{L}$ \\
3 & twisted & & $(1,1)_{0}$ & - & 0 & $\widetilde{\mathrm{U}(1)_{L}}$ \\
3 & $\mathrm{D} 7$ & & $(1,1)_{0}$ & $1_{0} \oplus 15_{0}$ & 0 & $\mathrm{SU}(4)_{F} \times \mathrm{U}(1)_{B}$ \\
\hline \hline
\end{tabular}

Table 11: Massless vector states for $\mathrm{SU}(N)$ w/ $2 \boxminus \oplus 4 \square$.

\section{4 $\operatorname{Sp}(2 N) \times \operatorname{Sp}(2 N)+\mathbf{1}$ bifundamental $+\mathbf{4}$ fundamental hypermultiplets}

The analysis of the $\operatorname{Sp}(2 N)_{1} \times \operatorname{Sp}(2 N)_{2}$ theory with hypermultiplet content $\left(\square_{1}, \square_{2}\right) \oplus 2 \square_{1} \oplus$ $2 \square 2$ proceeds in parallel with the $\mathrm{SU}(N)+2 \boxminus+4 \square$ theory, as they both share the same IIB background (with a few differences, discussed in the previous section, that serve to distinguish the two). Gukov and Kapustin [15] have analyzed this theory in detail, so we will just review their results and make a few remarks to complete and clarify them. 
The orientifold group and metric are the same as in Sect. 3.3 (see Eqs. (3.9) and (3.10)). As before, $\mathbb{Z}_{2}^{\text {orb }}$ fixes a six-dimensional hyperplane, while $\mathbb{Z}_{2}^{\text {orient }}$ fixes an eight-dimensional hyperplane. The fixed-point set becomes $S^{1} \cup S^{3} \subset S^{5}$ in the near-horizon limit. The global symmetry group, however, is different

$$
\mathrm{SU}(2)_{R} \times \mathrm{U}(1)_{R} \times \mathrm{SU}(2)_{L} \times \mathrm{SO}(4) \times \mathrm{SO}(4),
$$

and reflects the differences between the two cases. The $\mathrm{SO}(4) \times \mathrm{SO}(4)$ factor, which emerges in the brane configuration as the gauge group on the D7-branes, is the flavor symmetry of the fundamental hypermultiplets, two for each $\mathrm{Sp}(2 N)$ factor (the enhancement from $\mathrm{SU}(2)$ to $\mathrm{SO}(4)$ for self-conjugate representations was described in Sect. 3.2). The $\mathrm{SU}(2)_{L}$ factor is the flavor symmetry of the bifundamental hypermultiplet, as explained below. Note that the geometric part of the global symmetry, namely, $\mathrm{SU}(2)_{L} \times \mathrm{SU}(2)_{R} \times \mathrm{U}(1)_{R}$, is the same in both cases, a natural result given that both theories have the same geometric description

As in the previous section, the supergravity spectrum consists of three sectors:

\section{(a) Bulk sector:}

This sector consists of $A d S_{5} \times S^{5}$ supergravity states invariant under $\mathbb{Z}_{2}^{\text {orb }} \times \mathbb{Z}_{2}^{\text {orient }}$, and is identical to that in the $\mathrm{SU}(N)+2 \boxminus+4 \square$ theory, although the field theory interpretation of the resulting states obviously differs (see Table 12).

\section{(b) $\mathbb{Z}_{2}^{\text {orb }}$ twisted sector:}

The $\mathbb{Z}_{2}^{\text {orb }}$ twisted sector, supported on $A d S_{5} \times S^{1}$, consists of the KK reduction on $S^{1}$ of the six-dimensional $(2,0)$ tensor multiplet, modded out by $\mathbb{Z}_{2}^{\text {orient }}$. This is identical to the previous section, except that $\mathbb{Z}_{2}^{\text {orient }}$ acts on this sector with an additional minus sign.

(1) Scalar modes:

Recall that the triplet of scalars $(1,1 ; 1,3)$ gave rise upon KK reduction on $S^{1}$ to a tower of $\mathrm{SU}(2)_{R}$ triplets $(1,3)_{2 \ell}, \ell \geq 0$, with $m^{2}=\ell^{2}-4$. Taking into account the extra minus sign, $\Omega^{\prime}$

\footnotetext{
${ }^{5}$ In Ref. [15], only the Cartan subgroup $\mathrm{U}(1)_{L} \subset \mathrm{SU}(2)_{L}$ is considered, even though their tables contain all the states needed to form $\mathrm{SU}(2)_{L}$ multiplets. We will explicitly use the full $\mathrm{SU}(2)_{L}$ symmetry below, as this emphasizes the similarity with the $\mathrm{SU}(N)+2 \square+4 \square$ case.
} 
now acts as $e^{i \pi\left(q_{R} / 2+1\right)}=e^{i \pi(\ell+1)}$, leaving only states with $q_{R}=2 \bmod 4$ or $\ell=1 \bmod 2$ :

$$
\begin{aligned}
& \Delta=3 \quad(1,3)_{2}, \quad m^{2}=-3, \\
& \Delta=5 \quad(1,3)_{6}, \quad m^{2}=5, \\
& \vdots
\end{aligned}
$$

The scalars $(1,1 ; 1,1)$ gave rise to states $(1,1)_{2 \ell}, \ell \geq 2$, with $m^{2}=\ell^{2}-4 \ell$, which satisfy the constraint (3.6). $\Omega^{\prime}$ acts on these states as $e^{i \pi q_{R} / 2}=e^{i \pi \ell}$, which includes the extra minus sign coming from $\Omega(-1)^{F_{L}}$. Only states with $q_{R}=0 \bmod 4$, or $\ell=0 \bmod 2$, survive:

$$
\begin{aligned}
\Delta=2 & (1,1)_{4}, \quad m^{2}=-4, \\
4 & (1,1)_{8}, \quad m^{2}=0
\end{aligned}
$$

We include these states in Table 12 below.

(2) Vector modes:

The reduction on $S^{1}$ of the six-dimensional tensor field yields a massless vector state with $\mathrm{SU}(2)_{L} \times \mathrm{SU}(2)_{R} \times \mathrm{U}(1)_{R}$ quantum numbers $(1,1)_{0}$. This state, however, is projected out by $\Omega^{\prime}$, consistent with the fact that there is no global symmetry current other than the $\mathrm{SU}(2)_{L} \times$ $\mathrm{SU}(2)_{R} \times \mathrm{U}(1)_{R}$ currents from the bulk and the $\mathrm{SO}(4) \times \mathrm{SO}(4)$ currents from the D7-brane sector.

\section{(c) D7-brane sector:}

The D7-brane sector consists of the world-volume theory of 8 D7-branes with topology $A d S_{5} \times S^{3}$, modded out by $\mathbb{Z}_{2}^{\text {orb }}$. The $\mathbb{Z}_{2}^{\text {orb }}$ projection differs from that in the $\mathrm{SU}(N)+2 \boxminus+4 \square$ case, the corresponding projection matrix being - see Eq. (3.23):

$$
\gamma_{\theta 7}^{\mathrm{Sp} \times \mathrm{Sp}}=\left(\begin{array}{cc}
\mathbb{1}_{4} & 0 \\
0 & -\mathbb{1}_{4}
\end{array}\right)
$$

which breaks the $\mathrm{SO}(8)$ gauge group on the D7-branes to $\mathrm{SO}(4) \times \mathrm{SO}(4)$. Accordingly, the adjoint of $\mathrm{SO}(8)$ decomposes as $\mathbf{2 8} \rightarrow(6,1) \oplus(1,6) \oplus(4,4)$, where $(6,1) \oplus(1,6)$ is the adjoint representation of $\mathrm{SO}(4) \times \mathrm{SO}(4)$. In analogy with the $\mathrm{SU}(N)+2 \boxminus$ case, D7-brane excitations 
that are in odd representations of $\mathrm{SU}(2)_{L}$ transform in the $(6,1) \oplus(1,6)$ of the flavor group, while excitations in even representations of $\mathrm{SU}(2)_{L}$ transform in the $(4,4)$ of the flavor group. These results are summarized in Table 12.

\begin{tabular}{||c|c|c|c|c|c|c||}
\hline \hline$\Delta$ & Sector & $\mathrm{SU}(4)_{R}$ & $\left(d_{L}, d_{R}\right)_{q_{R}}$ & $\mathrm{SO}(4) \times \mathrm{SO}(4)$ & $m^{2}$ & CFT operator \\
\hline \hline 2 & bulk & $\mathbf{2 0}^{\prime}$ & $(1,1)_{4}$ & - & -4 & $\operatorname{tr} \Phi_{1}^{2}+\operatorname{tr} \Phi_{2}{ }^{2}$ \\
2 & bulk & $\mathbf{2 0}^{\prime}$ & $(3,3)_{0}$ & - & -4 & $\operatorname{tr}\left[B_{(i} J_{1} B_{j} J_{2}\right]$ \\
2 & twisted & & $(1,1)_{4}$ & - & -4 & $\operatorname{tr} \Phi_{1}^{2}-\operatorname{tr} \Phi_{2}{ }^{2}$ \\
2 & D7 & & $(1,3)_{0}$ & $(6,1) \oplus(1,6)$ & -4 & $Q_{1}^{[I} J_{1} Q_{1}^{K]} \oplus Q_{2}^{[I} J_{2} Q_{2}^{K]}$ \\
3 & bulk & $\mathbf{1 0}$ & $(1,3)_{2}$ & - & -3 & $\operatorname{tr} \lambda_{1}^{2}+\operatorname{tr} \lambda_{2}{ }^{2}$ \\
3 & twisted & & $(1,3)_{2}$ & - & -3 & $\operatorname{tr} \lambda_{1}^{2}-\operatorname{tr} \lambda_{2}^{2}$ \\
3 & D7 & & $(2,4)_{0}$ & $(4,4)$ & -3 & $Q_{1}^{I} J_{1} B_{i} J_{2} Q_{2}^{K}$ \\
4 & bulk & $\mathbf{1 0 5}$ & $(1,1)_{8}$ & - & 0 & $\operatorname{tr} \Phi_{1}^{4}+\operatorname{tr} \Phi_{2}{ }^{4}$ \\
4 & bulk & $\mathbf{1 0 5}$ & $(3,3)_{4}$ & - & 0 & $\operatorname{tr}\left[B_{(i} J_{1} \Phi_{1}{ }^{2} J_{2} B_{j}\right] \pm \operatorname{tr}\left[B_{(i} J_{1} \Phi_{2}{ }^{2} J_{2} B_{j}\right]$ \\
4 & bulk & $\mathbf{1 0 5}$ & $(5,5)_{0}$ & - & 0 & $\operatorname{tr}\left[J_{1} B_{(i} J_{2} B_{j} J_{1} B_{k} J_{2} B_{l)}\right]$ \\
4 & twisted & & $(1,1)_{8}$ & - & 0 & $\operatorname{tr} \Phi_{1}^{4}-\operatorname{tr} \Phi_{2}{ }^{4}$ \\
4 & D7 & & $(3,5)_{0}$ & $(6,1) \oplus(1,6)$ & 0 & $Q_{1}^{[I} J_{1} B_{(i} J_{2} B_{j)} J_{1} Q_{1}^{K]} \oplus Q_{2}^{[I} J_{2} B_{(i} J_{1} B_{j)} J_{2} Q_{2}^{K]}$ \\
\hline \hline
\end{tabular}

Table 12: Scalars with $\Delta \leq 4$ in chiral primary representations of $\operatorname{Sp}(2 N)_{1} \times \operatorname{Sp}(2 N)_{2} \mathrm{w} /\left(\square_{1}, \square_{2}\right) \oplus 2 \square_{1} \oplus 2 \square_{2}[15]$.

The last column of Table 12 lists the CFT operators that correspond to the supergravity states. The operators are written as products of fields in the perturbative $\operatorname{Sp}(2 N) \times \operatorname{Sp}(2 N)$ worldvolume theory, listed in Table 13. (It is difficult to specify the CFT operators unambiguously without explicitly writing all the gauge theory indices; instead, we adopt a slightly schematic notation, in which $J_{1}$ and $J_{2}$ denote the invariant antisymmetric tensor of $\operatorname{Sp}(2 N)$, acting on the indices of the first and second factor of the gauge group respectively.) 


\begin{tabular}{||c|c|c|c|c||}
\hline \hline$\Delta$ & $\mathrm{Sp}(2 N) \times \mathrm{Sp}(2 N)$ & $\mathrm{SU}(2)_{L} \times \mathrm{SU}(2)_{R} \times \mathrm{U}(1)_{R}$ & $\mathrm{SO}(4) \times \mathrm{SO}(4)$ & $\mathrm{CFT}$ field \\
\hline \hline$\frac{3}{2}$ & $($ adjoint,1) & $(1,2)_{1}$ & $(1,1)$ & $\lambda_{1}$ \\
$\frac{3}{2}$ & $(1$, adjoint $)$ & $(1,2)_{1}$ & $(1,1)$ & $\lambda_{2}$ \\
1 & $($ adjoint,1) & $(1,1)_{2}$ & $(1,1)$ & $\Phi_{1}$ \\
1 & $(1$, adjoint $)$ & $(1,1)_{2}$ & $(1,1)$ & $\Phi_{2}$ \\
1 & $(\square, \square)$ & $(2,2)_{0}$ & $(1,1)$ & $B_{i}$ \\
1 & $(\square, 1)$ & $(1,2)_{0}$ & $(4,1)$ & $Q_{1}^{I}$ \\
1 & $(1, \square)$ & $(1,2)_{0}$ & $Q_{2}^{I}$ \\
\hline \hline
\end{tabular}

Table 13 : $\mathrm{SU}(2)_{R}$ multiplets in perturbative $\operatorname{Sp}(2 N)_{1} \times \operatorname{Sp}(2 N)_{2} \quad \mathrm{w} / \quad\left(\square_{1}, \square_{2}\right) \oplus 2 \square_{1} \oplus 2 \square_{2}$

The $\mathrm{SU}(2)_{L}$ factor of the global symmetry corresponds to the flavor symmetry of the bifundamental hypermultiplet. The explanation is similar to that in the $\operatorname{Sp}(2 N)+\boxminus+4 \square$ case. The bifundamental hypermultiplet contains chiral superfields $B_{a_{1}}{ }_{2}$ in the $\left(\square_{1}, \bar{\square}_{2}\right)$ and $\tilde{B}^{a_{1}}{ }_{a_{2}}$ in the $\left(\square_{1}, \square_{2}\right)$ of $\operatorname{Sp}(2 N)_{1} \times \operatorname{Sp}(2 N)_{2}$. The piece of the $\mathcal{N}=2$ superpotential involving the first $\operatorname{Sp}(2 N)$ factor, say, is

$$
\tilde{B}^{a_{1}}{ }_{a_{2}} \Phi_{a_{1}}{ }^{b_{1}} B_{b_{1}}{ }^{a_{2}}
$$

where $\Phi_{a_{1}}{ }^{b_{1}}$ is a chiral superfield in the adjoint of $\operatorname{Sp}(2 N)_{1}$. We can define two chiral superfields $B_{1 a_{1} a_{2}} \equiv J_{a_{2} b_{2}} B_{a_{1}}{ }^{b_{2}}$ and $B_{2 a_{1} a_{2}} \equiv J_{a_{1} b_{1}} \tilde{B}^{b_{1}}{ }_{a_{2}}$, both transforming as $\left(\square_{1}, \square_{2}\right)$. In terms of these new fields the superpotential reads

$$
J^{a_{2} b_{2}} B_{2 a_{1} a_{2}} \widehat{\Phi}^{a_{1} b_{1}} B_{1 b_{1} b_{2}}
$$

where $\widehat{\Phi}^{a_{1} b_{1}}=J^{c_{1} a_{1}} \Phi_{c_{1}}{ }^{b_{1}}$ is symmetric in $a_{1}, b_{1}$. Since eq. (3.39) is odd under exchange $1 \leftrightarrow 2$, the superpotential actually possesses an $\mathrm{SU}(2)_{L}$ flavor symmetry acting on the doublet $B_{i}=$ $\left(B_{1}, B_{2}\right)$. A bare mass $\mathrm{m}$ (三 global mass) for the bifundamental hypermultiplet $\mathrm{m} B^{2}$ breaks $\mathrm{SU}(2)_{L} \rightarrow \mathrm{U}(1)_{L}$ 


\section{5 $\mathrm{SU}(N)+1$ antisymmetric +1 symmetric hypermultiplet}

We now move on to the models coming from the Type IIA $\mathrm{O}^{+}-\mathrm{O}^{-}$configuration. As in the $\mathrm{O} 6^{-}-\mathrm{O}^{-}$case, we obtain two theories with almost the same IIB description, namely $\mathrm{SU}(N)$ with symmetric and antisymmetric hypermultiplets, and $\operatorname{Sp}(2 N) \times \mathrm{SO}(2 N+2)$ with a bifundamental hypermultiplet. In the near-horizon supergravity description, both theories share the same bulk sector, and are only distinguished by the twisted sector of the $\mathbb{Z}_{2}^{\text {orb }}$ orbifold T-dual to the NS 5-branes. From Sect. 2.2, we recall that the orientifold group is

$$
G_{\text {orient }}=\mathbb{Z}_{2}^{\text {orb }}+\mathbb{Z}_{2}^{\text {orb }} \alpha \Omega^{\prime}
$$

where, as in previous cases, $\Omega^{\prime}=R_{45} \Omega(-1)^{F_{L}}$, and $\mathbb{Z}_{2}^{\text {orb }}=\left\{\mathbb{1}, \alpha^{2}\right\}$, with $\alpha^{2}=R_{6789}$. $G_{\text {orient }}$ is actually a $\mathbb{Z}_{4}$ group with elements $\left\{\mathbb{1}, \alpha \Omega^{\prime}, \alpha^{2}, \alpha^{3} \Omega^{\prime}\right\}$. Although (3.40) is superficially analogous to the orientifold group of the $\mathrm{O} 6^{-}-\mathrm{O}^{-}$models, the relevant fixed-point set of the $G_{\text {orient }}$ action reduces in this case to the six-dimensional hyperplane $x^{6}=x^{7}=x^{8}=x^{9}=0$ left fixed by $\mathbb{Z}_{2}^{\text {orb }}$, as the orientifold factor $\alpha \Omega^{\prime}$ only fixes the origin of the space transverse to the D3-branes, $x^{4}=x^{5}=x^{6}=x^{7}=x^{8}=x^{9}=0$, which does not give any contribution in the near-horizon limit. This is as expected, however, as we know that the T-dual of the $\mathrm{O}^{+}{ }_{-} \mathrm{O}^{-}$ configuration contains no D7-branes, which would be present had there been non-trivial fixed points associated with the $\Omega^{\prime}$ action.

In the near-horizon limit the space-time geometry is $A d S_{5} \times S^{5} / G_{\text {orient }}$, with the following metric for the compact space

$$
\begin{aligned}
& d \widetilde{\Omega}_{5}^{2}=d \theta_{1}^{2}+\cos ^{2} \theta_{1}\left(d \theta_{2}^{2}+\cos ^{2} \theta_{2} d \phi_{1}^{2}+\sin ^{2} \theta_{2} d \phi_{2}^{2}\right)+\sin ^{2} \theta_{1} d \phi_{3}^{2} \\
& \theta_{1,2} \sim \theta_{1,2}+\frac{\pi}{2}, \quad \phi_{1} \sim \phi_{1}+\frac{\pi}{2}, \quad \phi_{2} \sim \phi_{2}-\frac{\pi}{2}, \quad \phi_{3} \sim \phi_{3}+\pi .
\end{aligned}
$$

The isometry group of the above metric is a quotient of $\mathrm{U}(1)_{L} \times \mathrm{SU}(2)_{R} \times \mathrm{U}(1)_{R}$, where $\mathrm{U}(1)_{L} \subset \mathrm{SU}(2)_{L}$, which we can define as follows. $\alpha^{2}$ acts on $\mathrm{SU}(2)_{L}$ representations as $e^{i \pi J_{L}}$, with $J_{L} / 2$ the spin of the representation $\left(J_{L}=1\right.$ for the $\mathbf{2}, J_{L}=2$ for the $\mathbf{3}$, and so on), so it projects out even-dimensional representations, thereby breaking $\mathrm{SU}(2)_{L} \rightarrow \mathrm{SO}(3)_{L}$. $\alpha$ acts on $\mathrm{SO}(3)_{L}$ representations according to the weight of each state (or $\mathrm{U}(1)_{L} \subset \mathrm{SO}(3)_{L}$ charge $\left.q_{L}\right)$ as $e^{i \pi q_{L} / 2}$, where $q_{L}= \pm 2,0$ for the $\mathbf{3}, \pm 4, \pm 2,0$ for the $\mathbf{5}$, and so on, so that on $\operatorname{SO}(3)_{L}$ representations $\alpha$ acts as \pm 1 . This immediately suggests an alternative way to regard $G_{\text {orient }}$ 
that will prove useful in understanding the twisted sector of the spectrum, namely, to consider $G_{\text {orient }}$ as $\mathbb{Z}_{2}^{\text {orb }} \times\left(G_{\text {orient }} / \mathbb{Z}_{2}^{\text {orb }}\right)$ where the coset $G_{\text {orient }} / \mathbb{Z}_{2}^{\text {orb }} \sim\left\{\mathbb{1}, \alpha \Omega^{\prime}\right\}$. In other words, we mod out in two stages, first by $\mathbb{Z}_{2}^{\text {orb }}$, and then by $\alpha \Omega^{\prime}$. Both approaches give the same result for the bulk sector, while the second one makes the analysis of the twisted sector straightforward. Finally, $R_{45}$ acts on states with $\mathrm{U}(1)_{R}$ charge $q_{R}$ as $e^{i \pi q_{R} / 2}$. It is important to keep in mind when performing the projection that $G_{\text {orient }}$ has a single generator $\alpha \Omega^{\prime}$ (and will therefore constrain the allowed values of $\left.q_{L}+q_{R}\right)$. This differs from the projection in the $\mathrm{SU}(N)+2 \boxminus+4 \square$ or $\operatorname{Sp}(2 N) \times \operatorname{Sp}(2 N)$ cases, in which the orbifold and orientifold factors act independently, so that the $\mathrm{SU}(2)_{L}$ representation and $\mathrm{U}(1)_{R}$ charges of the states that survive the projection obey independent constraints.

The supergravity spectrum includes states from two sectors: (a) the bulk sector, consisting of $A d S_{5} \times S^{5}$ supergravity states invariant under $G_{\text {orient }}$, and (b) the $\mathbb{Z}_{2}^{\text {orb }}$ twisted sector, containing states twisted with respect to $\alpha^{2}$, and modded further by $\alpha \Omega^{\prime}$.

\section{(a) Bulk sector:}

The strategy to follow is essentially the same as in the $\mathrm{SU}(N)+2 \boxminus+4 \square$ case, but with a few differences that we will illustrate with two examples.

(1) Scalar modes:

Consider first the supergravity mode with $m^{2}=-4$ in the $\mathbf{2 0}^{\prime}$ of $\mathrm{SU}(4)_{R}$, which couples to a $\Delta=2$ operator on the boundary. Under the branching $\mathrm{SO}(6) \rightarrow \mathrm{SU}(2)_{L} \times \mathrm{SU}(2)_{R} \times \mathrm{U}(1)_{R}$, this representation decomposes as (see Table 5)

$$
\mathbf{2 0}^{\prime} \rightarrow(3,3)_{0}+(2,2)_{2}+(2,2)_{-2}+(1,1)_{4}+(1,1)_{-4}+(1,1)_{0} .
$$

$\mathbb{Z}_{2}^{\text {orb }}$ projects out all the states in even-dimensional representations of $\mathrm{SU}(2)_{L}$, leaving $(3,3)_{0}+$ $(1,1)_{4}+(1,1)_{-4}+(1,1)_{0}$. We now split the $\mathrm{SU}(2)_{L}$ multiplets according to their $\mathrm{U}(1)_{L}$ charge $q_{L}$, so that, for example, $(3,3)_{0} \rightarrow 3_{0}{ }^{ \pm 2}+3_{0}{ }^{0}$, where the superscript denotes $q_{L}$. We now keep only those states invariant under $\alpha \Omega^{\prime}$. Since $\alpha$ acts as $e^{i \pi q_{L} / 2}$ and the geometric part of $\Omega^{\prime}$ as $e^{i \pi q_{R} / 2}$, the surviving states must satisfy $q_{L}+q_{R}=0 \bmod 4$. This leaves

$$
3_{0}{ }^{0}, \quad 1_{ \pm 4}{ }^{0}, \quad 1_{0}{ }^{0}
$$


of which only $3_{0}{ }^{0}$ and $1_{4}{ }^{0}$ obey the constaint (3.6) and so correspond to chiral primary states of the CFT. $\left(1_{-4}{ }^{0}\right.$ is the complex conjugate of $1_{4}{ }^{0}$, and is an anti-chiral primary state.)

There are two modes with $m^{2}=-3$, in the $\mathbf{1 0}$ and the $\mathbf{5 0}$ of $\mathrm{SU}(4)_{R}$, which couple to $\Delta=3$ operators on the boundary. The $\mathbf{5 0}$ decomposes into

$$
\mathbf{5 0} \rightarrow(4,4)_{0}+(3,3)_{ \pm 2}+(2,2)_{ \pm 4}+(2,2)_{0}+(1,1)_{ \pm 6}+(1,1)_{ \pm 2}
$$

Projecting by $\alpha^{2}$ removes the even-dimensional representations of $\mathrm{SU}(2)_{L}$, leaving $(3,3)_{ \pm 2}+$ $(1,1)_{ \pm 6}+(1,1)_{ \pm 2}$. Breaking the resulting $\mathrm{SO}(3)_{L}$ to $\mathrm{U}(1)_{L}$, and projecting by $\alpha \Omega^{\prime}$ leaves states with $q_{L}+q_{R}=0 \bmod 4$, namely,

$$
3_{2}^{ \pm 2}, \quad 3-2^{ \pm 2}
$$

of which $3_{2}^{ \pm 2}$ are chiral primaries (while $3_{-2}{ }^{ \pm 2}$ are the complex conjugate anti-chiral primaries). The 10 decomposes into

$$
\mathbf{1 0} \rightarrow(1,3)_{2}+(3,1)_{-2}+(2,2)_{0}
$$

$\alpha^{2}$ removes $(2,2)_{0}$, while $\alpha \Omega^{\prime}$ keeps only those states with $q_{L}+q_{R}=2 \bmod 4$, taking into account the extra minus sign from the action of $\Omega(-1)^{F_{L}}$ on the ten-dimensional fields. This leaves $3_{2}{ }^{0}$ and $1_{-2}{ }^{0}$, of which only $3_{2}{ }^{0}$ is a chiral primary.

Proceeding in this way, one finds the results summarized in Table 14.

(2) Vector modes:

Start with the massless vector mode in the $\mathbf{1 5}$ of $\mathrm{SO}(6)$ (see Table 3), which couples to the $\Delta=3 \mathrm{SU}(4)_{R}$ current on the boundary. Given the branching

$$
\mathbf{1 5} \rightarrow(1,1)_{0}+(2,2)_{2}+(2,2)_{-2}+(3,1)_{0}+(1,3)_{0},
$$

we see that $\alpha^{2}$ projects out the $(2,2)_{ \pm 2}$, while the $\alpha \Omega^{\prime}$ projection leaves three massless vector states $1_{0}{ }^{0}, 3_{0}{ }^{0}, 1_{0}{ }^{0}$, which couple to the $\mathrm{U}(1)_{L} \times \mathrm{SU}(2)_{R} \times \mathrm{U}(1)_{R}$ currents of the boundary CFT.

(b) $\mathbb{Z}_{2}^{\text {orb }}$ twisted sector:

As in the $\mathrm{SU}(N)+2 \boxminus+4 \square$ or $\operatorname{Sp}(2 N) \times \operatorname{Sp}(2 N)$ cases, we start with the Type IIB theory on $\mathbb{C}_{6789}^{2} / \mathbb{Z}_{2}^{\text {orb }}$. The twisted sector consists of a $(2,0) d=6$ tensor multiplet, which we have to mod 
out by $\alpha \Omega^{\prime}$. The tensor multiplet is a singlet under $\mathrm{SU}(2)_{L}$ (see Table 8), and therefore insensitive to the action of $\alpha$, so we only have to project by $\Omega^{\prime}$, exactly as in the $\mathrm{SU}(N)+2 \boxminus+4 \square$ or $\operatorname{Sp}(2 N) \times \operatorname{Sp}(2 N)$ cases. As in those cases, there are two different ways to perform the projection [20]. The projection giving the $\operatorname{Sp}(2 N) \times \mathrm{SO}(2 N+2)$ twisted sector (which is the same as that for $\mathrm{Sp}(2 N) \times \mathrm{Sp}(2 N))$ has an extra minus sign relative to that giving the $\mathrm{SU}(N)+\boxminus+\square$ twisted sector (which is the same as that of $\mathrm{SU}(N)+2 \boxminus+4 \square$ ). We have come to the conclusion that the contribution to the supergravity spectrum of the $\mathbb{Z}_{2}^{\text {orb }}$ twisted sector for the $\mathrm{SU}(N)+\boxminus+$ theory is exactly the same as that of the $\mathrm{SU}(N)+2 \boxminus+4 \square$ theory, which we derived in section 3.3. (The interpretation in terms of field theory operators is of course different.)

\begin{tabular}{||c|c|c|c|c|c||}
\hline \hline$\Delta$ & Sector & $\mathrm{SU}(4)_{R}$ & $\left(d_{R}\right)_{q_{R}}{ }^{q_{L}}$ & $m^{2}$ & CFT operator \\
2 & bulk & $\mathbf{2 0}^{\prime}$ & $1_{4}{ }^{0}$ & -4 & $\operatorname{tr} \Phi^{2}$ \\
2 & bulk & $\mathbf{2 0}$ & $3_{0}{ }^{0}$ & -4 & $\tilde{A} A-\tilde{S} S$ \\
3 & bulk & $\mathbf{1 0}$ & $3_{2}{ }^{0}$ & -3 & $\operatorname{tr} \lambda^{2}$ \\
3 & bulk & $\mathbf{5 0}$ & $3_{2}{ }^{2}$ & -3 & $\tilde{S} \Phi A$ \\
3 & bulk & $\mathbf{5 0}$ & $3_{2}{ }^{2}$ & -3 & $\tilde{A} \Phi S$ \\
3 & twisted & & $1_{6}{ }^{0}$ & -3 & $\operatorname{tr} \Phi^{3}$ \\
& bulk & $\mathbf{1 0 5}$ & $5_{0}{ }^{4}$ & 0 & $(\tilde{S} A)^{2}$ \\
4 & bulk & $\mathbf{1 0 5}$ & $5_{0}{ }^{4}$ & 0 & $(\tilde{A} S)^{2}$ \\
4 & bulk & $\mathbf{1 0 5}$ & $5_{0}{ }^{0}$ & 0 & $(\tilde{A} A \pm \tilde{S} S)^{2}$ \\
4 & bulk & $\mathbf{1 0 5}$ & $3_{4}{ }^{0}$ & 0 & $\tilde{A} \Phi^{2} A-\tilde{S} \Phi^{2} S$ \\
4 & bulk & $\mathbf{1 0 5}$ & $1_{8}{ }^{0}$ & 0 & $\operatorname{tr} \Phi^{4}$ \\
4 & twisted & & $3_{4}{ }^{0}$ & 0 & $\tilde{A} \Phi^{2} A+\tilde{S} \Phi^{2} S$ \\
\hline \hline
\end{tabular}

Table 14: Scalars with $\Delta \leq 4$ in chiral primary representations of $\operatorname{SU}(N)$ w/ $\square \oplus 日$. The dimension of of the $\mathrm{SU}(2)_{R}$ representation is denoted by $d_{R}$, while $q_{R}, q_{L}$ denote the $\mathrm{U}(1)_{R}, \mathrm{U}(1)_{L}$ charges. 
The results are summarized in Table 14. The last column of Table 14 lists the CFT operators that we conjecture correspond to the supergravity states (see the discussion below). The operators are written as products of fields in the perturbative $\mathrm{SU}(N)+\square+\boxminus$ worldvolume theory, listed in Table 15.

\begin{tabular}{||c|c|c|c|c||}
\hline \hline$\Delta$ & $\mathrm{SU}(N)$ & $\mathrm{SU}(2)_{R} \times \mathrm{U}(1)_{R} \times \mathrm{U}(1)_{L}$ & $\widetilde{\mathrm{U}(1)_{L}}$ & $\mathrm{CFT}$ field \\
\hline \hline$\frac{3}{2}$ & adjoint & $2_{1}{ }^{0}$ & 0 & $\lambda$ \\
1 & adjoint & $1_{2}{ }^{0}$ & 0 & $\Phi$ \\
1 & $\boxminus$ & $2_{0}{ }^{1}$ & 1 & $A$ \\
1 & $\square$ & $2_{0}^{-1}$ & 1 & $S$ \\
\hline \hline
\end{tabular}

Table 15 : $\mathrm{SU}(2)_{R}$ multiplets in perturbative $\mathrm{SU}(N)$ w/ $\square \oplus \emptyset$

We recall that among the states from the $\mathbb{Z}_{2}^{\text {orb }}$ twisted sector is a massless vector with quantum numbers $1_{0}{ }^{0}$ that couples to a conserved current on the boundary. Let us denote the corresponding global (abelian) symmetry group by $\widetilde{\mathrm{U}(1)})_{L}$. We now consider how this $\widetilde{\mathrm{U}(1)_{L}}$, together with the $\mathrm{U}(1)$ symmetry from the bulk sector, acts on the fields of the theory.

The $\mathrm{SU}(N)+\boxminus+\square$ theory contains hypermultiplets $\{A, \tilde{A}\},\{S, \tilde{S}\}$ in the antisymmetric and symmetric representations of $\mathrm{SU}(N)$. The superpotential is

$$
\mathcal{W}=\operatorname{tr}(\tilde{A} \Phi A+\tilde{S} \Phi S)
$$

which possesses a $\mathrm{U}(1)_{A} \times \mathrm{U}(1)_{S}$ flavor symmetry acting as follows:

$$
\begin{array}{llll}
\multicolumn{2}{c}{\mathrm{U}(1)_{A}} & \mathrm{U}(1)_{S} \\
A \rightarrow e^{i \alpha} A & \tilde{A} \rightarrow e^{-i \alpha} \tilde{A} & A \rightarrow A & \tilde{A} \rightarrow \tilde{A} \\
S \rightarrow S & \tilde{S} \rightarrow \tilde{S} & S \rightarrow e^{i \beta} S & \tilde{S} \rightarrow e^{-i \beta} \tilde{S}
\end{array}
$$

We would like to identify $\mathrm{U}(1)_{L}$ and $\left.\widetilde{\mathrm{U}(1}\right)_{L}$ in terms of $\mathrm{U}(1)_{A}$ and $\mathrm{U}(1)_{S}$. We can give masses $m_{A} \tilde{A} A+m_{S} \tilde{S} S$ to both hypermultiplets without breaking $\mathrm{U}(1)_{A} \times \mathrm{U}(1)_{S}$. Defining $\mathrm{m}=$ 
$\left(m_{A}-m_{S}\right) / 2$ (global mass) and $\mu=\left(m_{A}+m_{S}\right) / 2$, we rewrite the mass term as

$$
\mathrm{m}(\tilde{A} A-\tilde{S} S)+\mu(\tilde{A} A+\tilde{S} S)
$$

Following the discussion of the $\mathrm{SU}(N)+2 \boxminus+4 \square$ theory, it seems reasonable to identify the $\mathrm{U}(1)_{L}$ and $\widetilde{\mathrm{U}(1)_{L}}$ generators, $T_{L}$ and $\widetilde{T}_{L}$, as

$$
T_{L}=T_{A}-T_{S}, \quad \widetilde{T}_{L}=\tilde{T}_{A}+\tilde{T}_{S}
$$

where $T_{A}$ and $T_{S}$ are the generators of $\mathrm{U}(1)_{A}$ and $\mathrm{U}(1)_{S}$. Thus, $\mathrm{U}(1)_{L} \times \widetilde{\mathrm{U}(1)_{L}}$ acts on the hypermultiplets as

$$
\begin{aligned}
& \mathrm{U}(1)_{L} \quad \widetilde{\mathrm{U}(1)_{L}} \\
& A \rightarrow e^{i \alpha} A \quad \tilde{A} \rightarrow e^{-i \alpha} \tilde{A} \quad A \rightarrow e^{i \beta} A \quad \tilde{A} \rightarrow e^{-i \beta} \tilde{A} \\
& S \rightarrow e^{-i \alpha} S \quad \tilde{S} \rightarrow e^{i \alpha} \tilde{S} \quad S \rightarrow e^{i \beta} S \quad \tilde{S} \rightarrow e^{-i \beta} \tilde{S}
\end{aligned}
$$

which we include in Table 15.

Further, based on analogy with the $\mathrm{SU}(N)+2 \boxminus+4 \square$ theory, it seems reasonable to identify the operator corresponding to deformation of the global mass, $\tilde{A} A-\tilde{S} S$, with the $3_{0}{ }^{0}$ supergravity operator in the bulk sector, and the operator corresponding to deformation of the average mass $\mu$ of the hypermultiplets, $\tilde{A} A+\tilde{S} S$, with the $3_{0}{ }^{0}$ supergravity operator in the twisted sector. This assignment, however, can only be regarded as conjectural. (Similar remarks hold for some of the other entries of Table 14.)

\section{6 $\mathrm{Sp}(2 N) \times \mathrm{SO}(2 N+2)+\mathbf{1}$ bifundamental hypermultiplet}

The analysis of the $\mathcal{N}=2 \mathrm{Sp}(2 N) \times \mathrm{SO}(2 N+2)$ theory with one bifundamental hypermultiplet proceeds in parallel with the previous one, as they both share the same IIB background (with a few differences that we have already discussed which serve to distinguish the two).

The orientifold group and metric are the same as in the previous case (see Eqs. (3.40) and (3.41)). As before, there is a six-dimensional hyperplane left fixed by the $G_{\text {orient }}$, which becomes $A d S_{5} \times S^{1}$ in the near-horizon limit.

The supergravity spectrum consists of two sectors: 


\section{(a) Bulk sector:}

This sector consists of $A d S_{5} \times S^{5}$ supergravity states invariant under the orientifold group (3.40), and is identical to that in the $\mathrm{SU}(N)+\square+\boxminus$ theory, although the field theory interpretation of the resulting states obviously differs (see Table 16).

(b) $\mathbb{Z}_{2}^{\text {orb }}$ twisted sector:

The $\mathbb{Z}_{2}^{\text {orb }}$ twisted sector, supported on $A d S_{5} \times S^{1}$, consists of the KK reduction on $S^{1}$ of the six-dimensional $(2,0)$ tensor multiplet, modded out by $\alpha \Omega^{\prime}$.

\begin{tabular}{|c|c|c|c|c|c|}
\hline$\Delta$ & Sector & $\mathrm{SU}(4)_{R}$ & $\left(d_{R}\right)_{q_{R}}^{q_{L}}$ & $m^{2}$ & CFT operator \\
\hline 2 & bulk & $20^{\prime}$ & $1_{4}^{0}$ & -4 & $\operatorname{tr} \Phi_{\mathrm{Sp}}^{2} \pm \operatorname{tr} \Phi_{\mathrm{SO}}^{2}$ \\
\hline 2 & bulk & $20^{\prime}$ & $3_{0}{ }^{0}$ & -4 & $\tilde{B} B$ \\
\hline 2 & twisted & & $1_{4}^{0}$ & -4 & $\operatorname{tr} \Phi_{\mathrm{Sp}}^{2} \mp \operatorname{tr} \Phi_{\mathrm{SO}}^{2}$ \\
\hline 3 & bulk & 10 & $3_{2}{ }^{0}$ & -3 & $\operatorname{tr} \lambda_{\mathrm{Sp}}^{2} \pm \operatorname{tr} \lambda_{\mathrm{SO}}^{2}$ \\
\hline 3 & bulk & 50 & $3_{2}^{2}$ & -3 & $B\left(J \Phi_{\mathrm{Sp}}\right) B \pm B \Phi_{\mathrm{SO}}(J B)$ \\
\hline 3 & bulk & 50 & $3_{2}^{-2}$ & -3 & $\tilde{B}\left(J \Phi_{\mathrm{Sp}}\right) \tilde{B} \pm \tilde{B} \Phi_{\mathrm{SO}}(J \tilde{B})$ \\
\hline 3 & twisted & & $3_{2}{ }^{0}$ & -3 & $\operatorname{tr} \lambda_{\mathrm{Sp}}^{2} \mp \operatorname{tr} \lambda_{\mathrm{SO}}^{2}$ \\
\hline 4 & bulk & 105 & $5_{0}^{4}$ & 0 & ВJBВJB \\
\hline 4 & bulk & 105 & $5_{0}^{-4}$ & 0 & $\tilde{B} J \tilde{B} \tilde{B} J \tilde{B}$ \\
\hline 4 & bulk & 105 & $5_{0}{ }^{0}$ & 0 & $(\tilde{B} B)^{2}$ \\
\hline 4 & bulk & 105 & $3_{4}{ }^{0}$ & 0 & $\tilde{B} \Phi_{\mathrm{Sp}}^{2} B \pm \tilde{B} \Phi_{\mathrm{SO}}^{2} B$ \\
\hline 4 & bulk & 105 & $1_{8}^{0}$ & 0 & $\operatorname{tr} \Phi_{\mathrm{Sp}}^{4} \pm \operatorname{tr} \Phi_{\mathrm{SO}}^{4}$ \\
\hline 4 & twisted & & $1_{8}^{0}$ & 0 & $\operatorname{tr} \Phi_{\mathrm{Sp}}^{4} \mp \operatorname{tr} \Phi_{\mathrm{SO}}^{4}$ \\
\hline
\end{tabular}

Table 16: Scalars with $\Delta \leq 4$ in chiral primary representations of $\operatorname{Sp}(2 N) \times \operatorname{SO}(2 N+2)$ w/ $(\square, \square)$

As $\alpha$ acts trivially on the tensor multiplet, which is an $\mathrm{SU}(2)_{L}$ singlet, we need only project by $\Omega^{\prime}$. This sector is therefore identical to that in the $\operatorname{Sp}(2 N) \times \operatorname{Sp}(2 N)$ theory (at least for 
large $N$ ). Recall that $\Omega^{\prime}$ acts on the twisted sector with an additional minus sign relative to the $\mathrm{SU}(N)+2 \boxminus+4 \square$ or $\mathrm{SU}(N)+\square+\boxminus$ theories.

The spectrum of scalars with $\Delta \leq 4$ in chiral primary representations is summarized in Table 16. The last column of Table 16 lists the CFT operators that correspond to the supergravity states. The operators are written as products of fields in the perturbative $\operatorname{Sp}(2 N) \times \mathrm{SO}(2 N+2)$ worldvolume theory, listed in Table 17. As in the previous section, in many cases there are several different CFT operators with the correct quantum numbers to match the supergravity states, and we do not even attempt to resolve the ambiguities.

\begin{tabular}{||c|c|c|c||}
\hline \hline$\Delta$ & $\mathrm{Sp}(2 N) \times \mathrm{SO}(2 N+2)$ & $\mathrm{SU}(2)_{R} \times \mathrm{U}(1)_{R} \times \mathrm{U}(1)_{L}$ & $\mathrm{CFT}$ field \\
\hline \hline$\frac{3}{2}$ & $($ adjoint, 1$)$ & $2_{1}{ }^{0}$ & $\lambda_{\mathrm{Sp}}$ \\
$\frac{3}{2}$ & $(1$, adjoint $)$ & $2_{1}{ }^{0}$ & $\lambda_{\mathrm{SO}}$ \\
1 & $($ adjoint, 1$)$ & $1_{2}{ }^{0}$ & $\Phi_{\mathrm{Sp}}$ \\
1 & $(1$, adjoint $)$ & $1_{2}{ }^{0}$ & $\Phi_{\mathrm{SO}}$ \\
1 & $(\square, \square)$ & $2_{0}{ }^{1}$ & $B$ \\
\hline \hline
\end{tabular}

Table $17: \mathrm{SU}(2)_{R}$ multiplets in perturbative $\mathrm{Sp}(2 N) \times \mathrm{SO}(2 N+2)$ w/ $(\square, \square)$.

\section{Acknowledgements}

We would like to thank E. Cortegoso for assistance with the figures. 


\section{References}

[1] J. Maldacena, The Large $N$ Limit of Superconformal Field Theories and Supergravity, Adv. Theor. Math. Phys. 2 (1998) 231, hep-th/9711200.

[2] S.S. Gubser, I.R. Klebanov, A.M. Polyakov, Gauge Theory Correlators from Non-Critical String Theory, Phys. Lett. B428 (1998) 105, hep-th/9802109.

[3] E. Witten, Anti De Sitter Space and Holography, Adv. Theor. Math. Phys. 2 (1998) 253, hep-th/9802150.

[4] O. Aharony, S.S. Gubser, J. Maldacena, H. Ooguri, Y. Oz, Large N Field Theories, String Theory and Gravity, Phys. Rept. 323 (2000) 183, hep-th/9905111.

[5] E.G. Gimon, J. Polchinski, Consistency Conditions for Orientifolds and D-Manifolds, Phys. Rev. D54 (1996) 1667, hep-th/9601038.

[6] M. Douglas, G. Moore, D-branes, Quivers, and ALE Instantons, hep-th/9603167.

[7] S. Kachru, E. Silverstein, 4d Conformal Field Theories and Strings on Orbifolds, Phys. Rev. Lett. 80 (1998) 4855, hep-th/9802183.

[8] A. Lawrence, N. Nekrasov, C. Vafa, On Conformal Theories in Four Dimensions, Nucl. Phys. B533 (1998) 199, hep-th/9803015.

[9] Y. Oz, J. Terning, Orbifolds of $A d S_{5} \times S^{5}$ and $4 d$ Conformal Field Theories, Nucl. Phys. B532 (1998) 163, hep-th/9803167.

[10] S. Gukov, Comments on $N=2$ AdS Orbifolds, Phys. Lett. B439 (1998) 23, hepth/9806180.

[11] Z. Kakushadze, Gauge Theories from Orientifolds and Large N Limit, Nucl. Phys. B529 (1998) 157, hep-th/9803214; On Large N Gauge Theories from Orientifolds, Phys. Rev. D58 (1998) 106003.

[12] E. Witten, Baryons and Branes in Anti de Sitter Space, JHEP 9807 (1998) 006, hepth/9805112.

[13] A. Fayyazuddin, M. Spalinski, Large N Superconformal Gauge Theories and Supergravity Orientifolds, Nucl. Phys. B535 (1998) 219, hep-th/9805096.

[14] O. Aharony, A. Fayyazuddin, J. Maldacena, The Large $N$ Limit of $\mathcal{N}=2,1$ Field Theories from Threebranes in F-theory, JHEP 9807 (1998) 013, hep-th/9806159.

[15] S. Gukov, A. Kapustin, New $N=2$ Superconformal Field Theories from $M / F$ Theory Orbifolds, Nucl. Phys. B545 (1999) 283, hep-th/9808175.

[16] M. Gremm, A. Kapustin, $N=1$ Theories, T-duality, and AdS/CFT Correspondence, JHEP 9907 (1999) 005, hep-th/9904050.

[17] N. Seiberg, E. Witten, Monopole Condensation, and Confinement in $N=2$ Supersymmetric Yang-Mills Theory, Nucl. Phys. B426 (1994) 19, erratum, ibid B430 (1994) 485, hep-th/9407087; Monopoles, Duality and Chiral Symmetry Breaking in $N=2$ Supersymmetric QCD, Nucl. Phys. B431 (1994) 484, hep-th/9408099. 
[18] E. Witten, Solutions of Four-Dimensional Field Theories Via M Theory, Nucl. Phys. B500 (1997) 3, hep-th/9703166.

[19] A.M. Uranga, Towards Mass Deformed $N=4 \mathrm{SO}(n)$ and $\operatorname{Sp}(k)$ Gauge Configurations, Nucl. Phys. B526 (1998) 241, hep-th/9803054.

[20] J. Park, A. M. Uranga, A Note on Superconformal $N=2$ Theories and Orientifolds, Nucl. Phys. B542 (1999) 139, hep-th/9808161.

[21] J. Erlich, A. Hanany, A. Naqvi, Marginal Deformations from Branes, JHEP 9903 (1999) 008, hep-th/9902118.

[22] I.P. Ennes, C. Lozano, S.G. Naculich, H.J. Schnitzer, Elliptic Models and M-Theory, hepth/9912133.

[23] J. Polchinski, M. Strassler, The String Dual of a Confining Four-dimensional Gauge Theory, hep-th/0003136.

[24] O. Aharony, A. Rajaraman, String Theory Duals for Mass-deformed $S O(N)$ and USp $(2 N)$ $\mathcal{N}=4 S Y M$ Theories, hep-th/0004151.

[25] A. Dabholkar, J. Park, Strings on Orientifolds, Nucl. Phys. B477 (1996) 701, hepth/9604178.

[26] E.G. Gimon, C.V. Johnson, K3 Orientifolds, Nucl. Phys. B477 (1996) 715, hepth/9604129.

[27] E. Witten, Toroidal Compactification without Vector Structure, JHEP 9802 (1998) 006, hep-th/9712028.

[28] J. Blum, K. Intriligator, Consistency Conditions for Branes at Orbifold Singularities, Nucl. Phys. B506 (1997) 223, hep-th/9705030.

[29] I. Brunner, A. Karch, Branes at Orbifolds versus Hanany Witten in Six Dimensions, JHEP 9803 (1998) 003, hep-th/9712143.

[30] A. Hanany, A. Zaffaroni, Branes and Six Dimensional Supersymmetric Theories, Nucl. Phys. B529 (1998) 180, hep-th/9712145.

[31] H.J. Kim, L.J. Romans, P. van Nieuwenhuizen, Mass Spectrum of Chiral Ten-dimensional $N=2$ Supergravity on $S^{5}$, Phys. Rev. D32 (1985) 389;

M. Günaydin, N. Marcus, The Spectrum of the $S^{5}$ Compactification of Chiral $\mathcal{N}=2$, $d=10$ Supergravity and the Unitarity Supermultiplets of U(2,2/4), Class. Quant. Grav. 2 (1985) L11.

[32] A. Sen, F-theory and Orientifolds, Nucl. Phys. B475 (1996) 562, hep-th/9605150.

[33] T. Banks, M. Douglas, N. Seiberg, Probing F-theory with Branes, Phys. Lett. B387 (1996) 278, hep-th/9605199.

[34] O. Aharony, C. Sonnenschein, S. Yankielowicz, S. Theisen, Field Theory Questions for String Theory Answers, Nucl. Phys. B493 (1997) 177, hep-th/9611222.

[35] M. Douglas, D. Lowe, J. Schwarz, Probing F-theory with Multiple Branes, Phys. Lett. B394 (1997) 297, hep-th/9612062. 
[36] S. Minwalla, Restrictions Imposed by Superconformal Invariance on Quantum Field Theories, Adv. Theor. Math. Phys. 2 (1998) 781, hep-th/9712074.

[37] A. Dabholkar, Lectures on Orientifolds and Duality, hep-th/9804208. 